\title{
Dust destruction by the reverse shock in the Cassiopeia A supernova remnant
}

\author{
Elisabetta R. Micelotta ${ }^{1,2, \star}$, Eli Dwek ${ }^{2}$, and Jonathan D. Slavin ${ }^{3}$ \\ 1 Institut d'Astrophysique Spatiale, UMR 8617, Université Paris-Sud, 91405 Orsay, France \\ 2 Observational Cosmology Lab, Code 665, NASA Goddard Space Flight Center, Greenbelt, MD 20771, USA \\ e-mail: elisabetta.micelotta@helsinki.fi \\ ${ }^{3}$ Harvard-Smithsonian Center for Astrophysics, 60 Garden Street, Cambridge, MA 02138, USA
}

Received 12 September 2015 / Accepted 6 February 2016

\begin{abstract}
Context. Core collapse supernovae (CCSNe) are important sources of interstellar dust, which are potentially capable of producing $1 M_{\odot}$ of dust in their explosively expelled ejecta. However, unlike other dust sources, the dust has to survive the passage of the reverse shock, generated by the interaction of the supernova blast wave with its surrounding medium. Knowledge of the net amount of dust produced by CCSNe is crucial for understanding the origin and evolution of dust in the local and high-redshift Universe.

Aims. We identify the dust destruction mechanisms in the ejecta and derive the net amount of dust that survives the passage of the reverse shock.

Methods. We use analytical models for the evolution of a supernova blast wave and of the reverse shock with special application to the clumpy ejecta of the remnant of Cassiopeia A (Cas A). We assume that the dust resides in cool oxygen-rich clumps, which are uniformly distributed within the remnant and surrounded by a hot X-ray emitting plasma (smooth ejecta), and that the dust consists of silicates $\left(\mathrm{MgSiO}_{3}\right)$ and amorphous carbon grains. The passage of the reverse shock through the clumps gives rise to a relative gas-grain motion and also destroys the clumps. While residing in the ejecta clouds, dust is processed via kinetic sputtering, which is terminated either when the grains escape the clumps or when the clumps are destroyed by the reverse shock. In either case, grain destruction proceeds thereafter by thermal sputtering in the hot shocked smooth ejecta.

Results. We find that 11.8 and 15.9 percent of silicate and carbon dust, respectively, survive the passage of the reverse shock by the time the shock has reached the centre of the remnant. These fractions depend on the morphology of the ejecta and the medium into which the remnant is expanding, as well as the composition and size distribution of the grains that formed in the ejecta. Results will therefore differ for different types of supernovae.
\end{abstract}

Key words. dust, extinction - ISM: supernova remnants - shock waves - supernovae: general - supernovae: individual: Cassiopeia A

\section{Introduction}

Core collapse supernovae (CCSNe) are major producers of heavy elements and drive the chemical enrichment in galaxies. The high densities and preponderance of refractory elements in their ejecta provide the necessary conditions for the formation and growth of dust. Dust of supernova (SN) origin was found in meteorites (see reviews in Clayton \& Nittler 2004; Zinner 2008), and its presence was also inferred from their infrared (IR) and sub-millimeter emission in young $\mathrm{SNe}$, such as SN 1987A (Moseley et al. 1989; Dwek et al. 1992; Wooden 1997; Bouchet et al. 2004; Indebetouw et al. 2014; Matsuura et al. 2015, and references therein), and in young unmixed supernova remnants (SNRs) such as Cassiopeia A (Cas A; Lagage et al. 1996; Rho et al. 2008; Dunne et al. 2009; Barlow et al. 2010; Arendt et al. 2014) or the Crab Nebula (Hester 2008; Gomez et al. 2012; Temim \& Dwek 2013). The observations of SNRs suggest that the typical yield of dust is about $0.1 M_{\odot}$, with the largest inferred dust mass of $\sim 0.5 M_{\odot}$ in SN 1987A (Matsuura et al. 2015). Theoretical models suggest that refractory elements precipitate very efficiently from the gas phase, giving a dust yield of $0.1-0.5 M_{\odot}$ for a typical $25 M_{\odot}$ progenitor star (Todini \& Ferrara 2001; Schneider et al. 2004; Cherchneff \& Dwek 2010; Nozawa et al. 2010). A review of

\footnotetext{
* Present address: Department of Physics, PO Box 64, University of
} Helsinki, 00014 Helsinki, Finland. the production of dust in galaxies was presented by Gall et al. (2011c).

Supernovae also destroy dust during the remnant phase of their evolution. Most recent calculations show that in the local solar neighbourhood SNRs destroy more dust than is produced by $\mathrm{SNe}$ and asymptotic giant branch (AGB) stars combined (Bocchio et al. 2014; Slavin et al. 2015, and references therein). Similar conclusions were reached for the Magellanic Clouds (Temim et al. 2015). This imbalance between the production and destruction rate also prevails in local and high-redshift galaxies; this suggests that dust may need to reconstitute by accretion in the dense phases of the interstellar medium (ISM; Dwek \& Scalo 1980; Valiante et al. 2011; Dwek et al. 2011; Gall et al. 2011a,b). The problem becomes more acute in the very high redshift Universe $(z \gtrsim 6)$ where large amounts of dust have been detected $\left(\gtrsim 10^{7} M_{\odot}-\right.$ Bertoldi et al. 2003; Watson et al. 2015), but AGB stars have not yet evolved to form dust. If they are to be the sole source of dust in the early Universe Dwek \& Cherchneff (2011), SNe must then produce $\sim 1 M_{\odot}$ of dust. Supernovae are net producers of interstellar dust in galaxies with metallicities below $\sim 0.002$ only, and $\mathrm{SNe}$ are capable of producing a significant amount of dust with a dust yield of $\sim 0.1 M_{\odot} / \mathrm{SN}$ without resorting to the need of grain growth in the ISM (Dwek et al. 2014).

Calculated dust yields, and dust yields that are observationally inferred for very young $\mathrm{SNe}$, do not reflect the net amount 
of dust that is ultimately injected by SNe into the ISM. The pressure of the ISM gas that is shocked by the expanding SN blast wave generates a reverse shock that propagates through the expanding ejecta (McKee 1974; Truelove \& McKee 1999), partially destroying the newly formed dust by sputtering (Dwek 2005; Bianchi \& Schneider 2007; Nath et al. 2008; Nozawa et al. 2007; Silvia et al. 2010, 2012; Micelotta \& Dwek 2013; Biscaro \& Cherchneff 2014, 2016). However, because of the complexity of the problem, the total amount of mass destroyed is still unclear.

In this paper we calculate the mass of dust destroyed in the most extensively studied SNR, Cas A. We illustrate the relative importance of the many physical processes in the shocked ejecta by generalizing the analytical model of Truelove \& McKee (1999, hereafter TM99) to describe the evolution of the forward and reverse shock in the remnant. We adopt a set of parameters that reproduce the dynamics and the density and temperature profile of the Cas A ejecta. The reverse shock has partially propagated through its ejecta (Gotthelf et al. 2001) heating the $\mathrm{SN}$-condensed dust, which gives rise to observed mid- to far-IR emission from the remnant (Ennis et al. 2006; Rho et al. 2008; Arendt et al. 2014). The mass of dust inferred from these observations is about $0.1 M_{\odot}$ (Arendt et al. 2014). We assume that all the dust is initially in the clumps, and that there is no dust in the interclump region. The mass, composition, and size distribution of the dust that survives the passage of the reverse shock and is injected into the ISM is the subject of this publication.

The paper is organized as follows. We first present our generalization of the analytical model of TM99 to the case of nonuniform ejecta expanding into a non-uniform ambient medium (Sect. 2), and in Sect. 3 we provide the specific results of this model for Cas A. We assume that the dynamics of the reverse shock is unaffected by the presence of clumps (clouds) in the ejecta, and adopt the parameters of Sutherland \& Dopita (1995) to describe their density. The density contrast between the clumps and the smooth ejecta determines the velocity of the reverse shock that propagates into the clumps.

The reverse shock propagating through the clumps has low velocity compared to that of the reverse shock propagating through the smooth ejecta. The passage of the reverse shock causes a relative dust-gas motion and subjects the dust to kinetic sputtering. The amount of grain destruction depends on the initial velocity of the grains, and the mass column density they traverse through the clump, which in turn depends on both the size of the grains and their position in the cloud. In Sect. 4 we present the formalism that we use to investigate the effect of kinetic sputtering inside the clumps.

Kinetic sputtering is terminated either when the grains escape the clump, or when the clump is destroyed by crushing and evaporation. At the end of the kinetic sputtering process, the grains are injected into the ambient hot gas. The average thermal kinetic energy of the gas exceeds the kinetic energy of the grains, and grain destruction proceeds predominantly by thermal sputtering. In Sect. 5 we study the time it takes the dust to travel through the cloud, and compare this to the cloud crushing and evaporation timescales.

Any dust escaping the clumps is subjected to thermal sputtering in the hot SN cavity. Conditions in the cavity evolve with time as the density and temperature of the X-ray emitting gas decrease by expansion and radiative cooling. The destruction of dust in this evolving environment is presented in Sect. 6. We adopt silicates in the form of $\mathrm{MgSiO}_{3}$ and amorphous carbon as the main dust components in the ejecta, and assume that their initial size distribution is characterized by a power law in grain radii. Section 7 presents our Monte Carlo approach to evaluate kinetic and thermal sputtering, together with our results in terms of the net amount and mass distribution as a function of grain size of the surviving dust in Cas A. A discussion of the astrophysical significance of our findings is presented in Sect. 8, and our results are summarized in Sect. 9.

\section{Dynamics}

Cas A is a young SNR ( 333 yr in 2004) currently in an intermediate stage between the two non-radiative phases of supernova remnants evolution (Laming \& Hwang 2003): the ejecta-dominated phase (ED), when the mass of the ejecta is dominant with respect to the swept-up circumstellar material, and the Sedov-Taylor phase (ST), which starts as the remnant has swept-up an amount of circumstellar material comparable with the mass of the stellar ejecta.

To describe the dynamical evolution of the SNR through the ED and ST stages, we refer to the analytical treatment of TM99. This seminal work focused on the evolution of supernova ejecta expanding into an uniform density ambient medium, emphasising the dependence of the ED stage on the ejecta parameters and providing analytical expressions that smoothly merge the blastwave and reverse shock solutions between the ED and ST stages. We generalize their treatment to general power-law ambient media (described by an index $s: \rho(r)=\rho_{s} r^{-s}$ ), following the indications provided in their Appendix A. Then, referring to the work of Laming \& Hwang (2003), we consider the specific case of $s=2$ appropriate for Cas A, i.e. ejecta expanding into a presupernova steady stellar wind (van den Bergh 1971; Chevalier \& Fransson 1994; Laming \& Hwang 2003, and references therein).

\subsection{Characteristic scales}

As shown by TM99, in the problem considered here the initial conditions introduce three independent dimensional parameters: the ejecta energy, $E$, ejecta mass, $M_{\mathrm{ej}}$ and normalization parameter for the ambient density, $\rho_{s}$. These three dimensional parameters can be combined in a unique way to define characteristic scales of length, time, and mass as follows:

$$
\begin{aligned}
& R_{\mathrm{ch}} \equiv M_{\mathrm{ej}}^{1 /(3-s)} \rho_{s}^{-1 /(3-s)}, \\
& t_{\mathrm{ch}} \equiv E^{-1 / 2} M_{\mathrm{ej}}^{(5-s) / 2(3-s)} \rho_{s}^{-1 /(3-s)}, \\
& M_{\mathrm{ch}} \equiv M_{\mathrm{ej}} .
\end{aligned}
$$

Additional scales can be derived from the following base set:

$v_{\mathrm{ch}} \equiv R_{\mathrm{ch}} / t_{\mathrm{ch}}=\left(E / M_{\mathrm{ej}}\right)^{1 / 2}$

$T_{\mathrm{ch}} \equiv \frac{3}{16} \frac{\mu}{k_{\mathrm{B}}} v_{\mathrm{ch}}^{2}$,

where $\mu$ is the mean mass per particle and $k_{\mathrm{B}}$ is the Boltzmann constant.

We adopt $s=2$, which corresponds to ejecta expanding into a pre-supernova steady stellar wind. In this case, $\rho_{s}=\rho_{2}=$ $n_{0} R_{\mathrm{b} 0}^{2}$, where $R_{\mathrm{b} 0}$ is the blast-wave radius at a given time and $n_{0}$ is the circumstellar density at $R_{\mathrm{b} 0}$. We can write

$R_{\mathrm{ch}}=40.74 M_{\mathrm{ej}}\left(n_{0} R_{\mathrm{b} 0}^{2}\right)^{-1} \mathrm{pc}$

$t_{\mathrm{ch}}=5633 E^{-1 / 2} M_{\mathrm{ej}}^{3 / 2}\left(n_{0} R_{\mathrm{b} 0}^{2}\right)^{-1} \mathrm{yr}$, 
with $M_{\mathrm{ej}}$ in solar masses, $E$ in units of $10^{51} \mathrm{erg}, n_{0}$ in $\mathrm{H}$ atoms per cubic centimeter, and $R_{\mathrm{b} 0}$ in parsecs.

Following TM99, we perform our calculations using the dimensionless starred variables defined as $R^{*} \equiv R / R_{\mathrm{ch}}, t^{*} \equiv t / t_{\mathrm{ch}}$, $v^{*} \equiv v / v_{\mathrm{ch}}$, etc. The results are presented in usual units for clarity.

\subsection{Initial conditions}

We assume that the $\mathrm{SN}$ ejecta initially expands homologously with a maximum ejecta velocity $v_{\mathrm{ej}}$, and velocity profile $v(r) \sim$ $r / t$ for $r<R_{\mathrm{ej}}$, where $R_{\mathrm{ej}} \equiv v_{\mathrm{ej}} t$ is the free-expansion radius of the outer layer of the ejecta if no ambient medium is present. The density is given by the following expression (Eq. (A.1) in TM99):

$\rho(r, t)= \begin{cases}\rho_{\mathrm{ej}}(v, t) \equiv \frac{M_{\mathrm{ej}}}{v_{\mathrm{ej}}^{3}} f\left(\frac{v}{v_{\mathrm{ej}}}\right) t^{-3} & r<R_{\mathrm{ej}} \\ \rho_{s} r^{-s} & r>R_{\mathrm{ej}},\end{cases}$

where $\rho_{s}$ is a normalization constant and the condition $s<3$ is required to ensure a finite ambient mass. In the initial ejecta density distribution, the term $t^{-3}$ accounts for the free expansion of the ejecta before encountering the circumstellar medium, while the time-independent shape of the density distribution is described by the structure function $f\left(v / v_{\mathrm{ej}}\right)$. We consider a powerlaw structure function expressed as

$f(w)= \begin{cases}f_{0} & 0 \leq w \leq w_{\text {core }} \\ f_{n} w^{-n} & w_{\text {core }} \leq w \leq 1,\end{cases}$

where $w \equiv v / v_{\text {ej }}, w_{\text {core }}=v_{\text {core }} / v_{\text {ej }}$ and $v_{\text {core }}$ is the velocity of the ejecta at the boundary between an inner uniform core region and an external power-law envelope region characterized by the index $n$. Following Chevalier \& Oishi (2003) and Laming $\&$ Hwang (2003), we adopt for Cas A the value $n=9$, which provides: i) the correct ratio between the forward and reverse shock radii; ii) the correct relationship between the forward shock and free-expansion rates; and iii) a more plausible ejecta mass of $2 M_{\odot}$. As discussed by TM99, a power-law ejecta envelope with index $n>5$ requires the presence of a core in order for $M_{\mathrm{ej}}$ to be finite.

The parameter $f_{0}$ can be determined imposing the continuity of $f(w)$ in $w_{\text {core }}$, i.e.

$f_{0}=f_{n} w_{\text {core }}^{-n}$.

The value of $f_{n}$ results from the condition that the integral of the mass must equal $M_{\mathrm{ej}}$, i.e.

$f_{n}=\frac{3}{4 \pi}\left[\frac{1-n / 3}{1-(n / 3) w_{\text {core }}^{3-n}}\right]$.

We can introduce the following dimensionless ratio:

$\frac{E}{(1 / 2) M_{\mathrm{ej}} v_{\mathrm{ej}}^{2}}=\left(\frac{3-n}{5-n}\right)\left(\frac{w_{\mathrm{core}}^{-(5-n)}-n / 5}{w_{\text {core }}^{-(3-n)}-n / 3}\right) w_{\mathrm{core}}^{2} \equiv \bar{\alpha}$.

If $n>5$, in the limit $w_{\text {core }} \rightarrow 0$, from Eq. (8) we obtain

$\rho_{\mathrm{ej}}(v, t) \equiv \frac{M_{\mathrm{ej}}}{v_{\text {core }}^{3}}\left(\frac{3}{4 \pi} \frac{n-3}{n}\right)\left(\frac{v}{v_{\text {core }}}\right)^{-n} t^{-3}$

while Eq. (12) becomes

$\frac{E}{(1 / 2) M_{\mathrm{ej}} v_{\text {core }}^{2}}=\frac{3}{5}\left(\frac{n-3}{n-5}\right)$.
From this, we can derive the expression for $v_{\text {core }}$ as follows:

$v_{\text {core }}=\left(\frac{10}{3} \frac{n-5}{n-3}\right)^{1 / 2}\left(\frac{E}{M_{\mathrm{ej}}}\right)^{1 / 2}$.

Remembering that $v_{\mathrm{ch}}=\left(E / M_{\mathrm{ej}}\right)^{1 / 2}$ and that $v^{*} \equiv v / v_{\mathrm{ch}}$ we then obtain

$v_{\text {core }}^{*}=\left(\frac{10}{3} \frac{n-5}{n-3}\right)^{1 / 2}$.

For the density profile of the ejecta core, Eqs. (8) and (9) yield

$\rho_{\mathrm{ej}}(t)=\frac{M_{\mathrm{ej}}}{v_{\mathrm{ej}}^{3}} f_{0} t^{-3}$.

To derive $f_{0}$, we impose the continuity between Eqs. (17) and (13), which is valid for the ejecta envelope, at the boundary between the inner uniform core and the external power-law envelope, where $v=v_{\text {core }}$. For $v_{\text {core }}$, we use Eq. (15). Following this procedure we obtain

$f_{0}=\left(\frac{v_{\mathrm{ej}}}{v_{\text {core }}}\right)^{3}\left(\frac{3}{4 \pi} \frac{n-3}{n}\right)$,

which substituted in Eq. (17) provides the following expression for the density profile of the ejecta core:

$\rho_{\mathrm{ej}}(t)=\frac{M_{\mathrm{ej}}}{v_{\mathrm{core}}^{3}}\left(\frac{3}{4 \pi} \frac{n-3}{n}\right) t^{-3}$.

It is convenient to characterize the layers of the ejecta by a dimensionless parameter $\alpha$, defined by $v(0) \equiv \alpha v_{\mathrm{ej}}(\alpha \leq 1)$, where $v(0)$ is the initial velocity of the layer with respect to the unperturbed ISM. The outer layer of the ejecta expands at velocity $v_{\mathrm{ej}}$ and is therefore characterized by $\alpha=1$, whereas the innermost layer has a value of $\alpha=0$. A layer $\alpha$ encounters the reverse shock at time $t_{\mathrm{r}}$ when its radius is equal to the position of the reverse shock, i.e. when $\alpha v_{\mathrm{ej}} t_{\mathrm{r}}=R_{\mathrm{r}}\left(t_{\mathrm{r}}\right)$. From this, we obtain

$\alpha=\frac{R_{\mathrm{r}}}{v_{\mathrm{ej}} t_{\mathrm{r}}}=\frac{R_{\mathrm{r}}}{R_{\mathrm{ej}}}$.

The relation between $t_{\mathrm{r}}$ and the parameter $\alpha$ is shown in Fig. 1, while the ejecta density from Eqs. (13) and (19), but expressed as a function of $\alpha$, is reported in Fig. 2. The mass density of the Cas A ejecta in $2004(\alpha=0.33)$ is indicated by the horizontal line and corresponds to a number density of $0.23 \mathrm{~cm}^{-3}$. This value is consistent with the average density of $\sim 0.25 \mathrm{~cm}^{-3}$ estimated by Morse et al. (2004) for the smooth X-ray emitting ejecta in Cas A.

\subsection{Final equations for the supernova shocks}

To derive the equations describing the evolution of the blast wave and of the reverse shock, we use the treatment from Laming \& Hwang (2003) as a starting point. However, to develop our calculations we fully evaluated the implications of the assumption that $w_{\text {core }} \rightarrow 0$ (instead of adopting $w_{\text {core }} \rightarrow 1$ as by Laming \& Hwang 2003).

In this section, we report the final expressions that we use in the rest of our study. For more details about the derivation of these equations, see Appendix A.

We introduce the quantity $\phi$, defined as the ratio of the pressures behind the reverse and blastwave shocks (McKee 1974). 


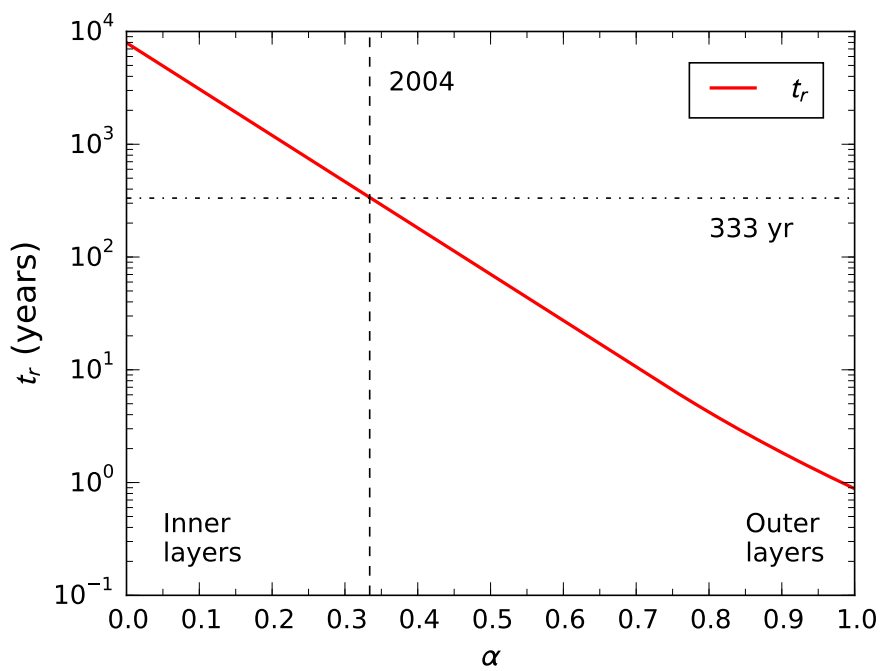

Fig. 1. Time, $t_{\mathrm{r}}$, after the explosion at which an ejecta layer characterized by the parameter $\alpha$ encounters the reverse shock. The outer layer has the value $\alpha=1$, while the inner one has $\alpha=0$. The vertical line indicates the value $\alpha=0.33$, corresponding to the ejecta layer which encounters the reverse shock in 2004, and of course intersects the red solid curve at the time corresponding to the age of Cas A in 2004, i.e. $t_{\mathrm{r}}=333 \mathrm{yr}$ (indicated by the horizontal line).

The lead factor $\ell$ is defined as the ratio of the radii of the forward shock to the reverse shock:

$\ell(t) \equiv \frac{R_{\mathrm{b}}(t)}{R_{\mathrm{r}}(t)}$.

Following TM99, we make the approximations $\phi(t) \cong \phi(0) \equiv$ $\phi_{\mathrm{ED}}$ and $\ell(t) \cong \ell(0) \equiv \ell_{\mathrm{ED}}$. The subscript "ED" refers to the ejecta-dominated phase of the evolution of the supernova remnant. With these approximations, from Eq. (21) we obtain $R_{\mathrm{r}}=R_{\mathrm{b}} / \ell_{\mathrm{ED}}$. The quantities $\phi_{\mathrm{ED}}$ and $\ell_{\mathrm{ED}}$ are well fitted by the following expressions (Hwang \& Laming 2011):

$\phi_{\mathrm{ED}}=[0.65-\exp (-n / 4)] \sqrt{1-\frac{s}{3}}$,

$\ell_{\mathrm{ED}}=1+\frac{8}{n^{2}}+\frac{0.4}{4-s}$.

During the ejecta-dominated phase, the reverse shock propagates through the ejecta envelope before reaching the ejecta core. The blast-wave radius during the initial envelope phase is given by the following expression:

$R_{\mathrm{b}}^{*}=\left\{v_{\mathrm{core}}^{*^{n-3}} \frac{(3-s)^{2}}{n(n-3)} \frac{3}{4 \pi} \frac{\ell_{\mathrm{ED}}^{n-2}}{\phi_{\mathrm{ED}}}\right\}^{\frac{1}{n-s}} t^{* \frac{n-3}{n-s}}$,

while the velocity of the forward shock is written as

$v_{\mathrm{b}}^{*}=\frac{n-3}{n-s} \frac{R_{\mathrm{b}}^{*}}{t^{*}}$.

This comes directly from Eq. (24) using the definition of velocity, $v_{\mathrm{b}}^{*}=\mathrm{d} R_{\mathrm{b}}^{*} / \mathrm{d} t^{*}$.

During the core phase, when the reverse shock propagates through the ejecta core, the blast-wave radius is given by

$$
\begin{aligned}
R_{\mathrm{b}}^{*}= & \left\{\left[\left(\frac{(3-s)^{2}}{n(n-3)} \frac{3}{4 \pi} \frac{l_{\mathrm{ED}}^{n-2}}{\phi_{\mathrm{ED}}}\right)\left(v_{\mathrm{core}}^{*} t_{\mathrm{conn}}^{*}\right)^{n-3}\right]^{\frac{5-s}{2(n-s)}}\right. \\
& \left.+\xi_{s}^{1 / 2}\left(t^{*}-t_{\mathrm{conn}}^{*}\right)\right\}^{\frac{2}{5-s}}
\end{aligned}
$$

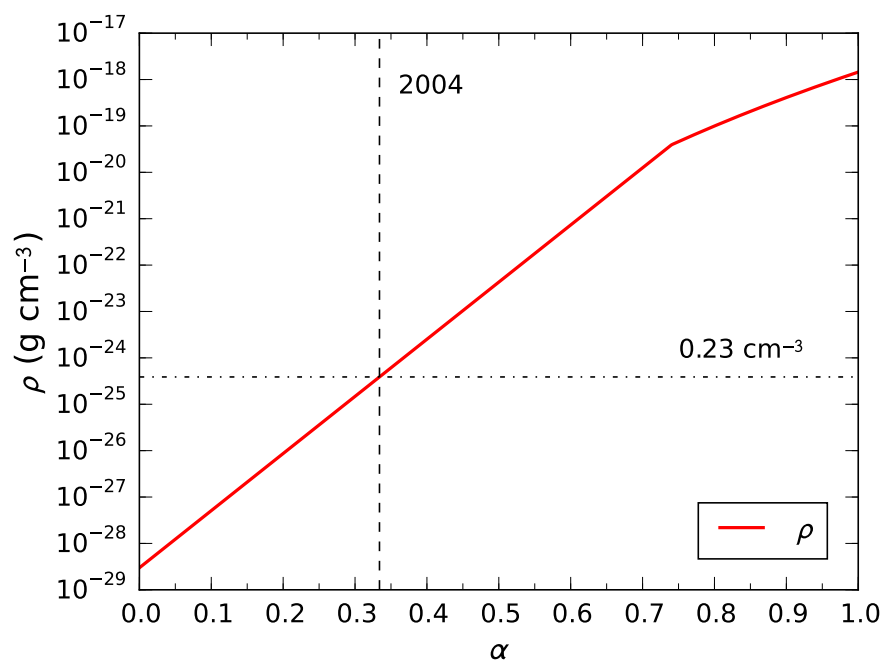

Fig. 2. Density structure of the ejecta calculated from Eq. (13) but expressed as a function of the parameter $\alpha$. Vertical line as in Fig. 1. The horizontal line indicates the mass density of the Cas A smooth ejecta in 2004 and the label indicates the corresponding number density.

and the blast-wave velocity is written as

$v_{\mathrm{b}}^{*}=\frac{2}{5-s} \xi_{s}^{1 / 2} R_{\mathrm{b}}^{\frac{s-3}{2}}$

with

$\xi_{s}=\frac{(5-s)(10-3 s)}{8 \pi}$

and

$t_{\text {conn }}^{*}=\left(\frac{n-3}{n-s} \sqrt{2 \pi \frac{5-s}{10-3 s}}\right)^{\frac{2(n-s)}{(5-n)(3-s)}}\left(v_{\text {core }}^{*} \ell_{\mathrm{ED}}\right)^{\frac{(n-s)(5-s)}{(5-n)(3-s)}} t_{\text {core }}^{\frac{5-s}{5-n}}$,

where $v_{\text {core }}^{*}$ comes from Eq. (16) and $t_{\text {core }}^{*}$ is the time at which the reverse shock hits the ejecta core, i.e.

$t_{\mathrm{core}}^{*}=\left[\frac{\ell_{\mathrm{ED}}^{s-2}}{\phi_{\mathrm{ED}}} \frac{3}{4 \pi} \frac{(3-s)^{2}}{n(n-3)}\right]^{1 /(3-s)} \frac{1}{v_{\mathrm{core}}^{*}}$.

Equations (24) and (25) are valid for $t^{*} \leq t_{\text {conn }}^{*}$, while Eqs. (26) and (27) are valid for $t^{*}>t_{\text {conn }}^{*}$. The reason for the introduction of the time $t_{\text {conn }}^{*}$, its definition and derivation can be found in Appendix A.

The equations for the reverse shock are the following. During the envelope phase, i.e. for $t^{*} \leq t_{\text {core }}^{*}$, the reverse shock radius is written as

$R_{\mathrm{r}}^{*}=\frac{R_{\mathrm{b}}^{*}}{\ell_{\mathrm{ED}}}$

and the reverse shock velocity (in the frame of the unshocked ejecta) is written as

$v_{\mathrm{r}}^{*}=\frac{3-s}{n-3} \frac{v_{\mathrm{b}}^{*}}{\ell_{\mathrm{ED}}}$.

During the core phase, i.e. for $t^{*}>t_{\text {core }}^{*}$, we have for the reverse shock radius the following expression:

$R_{\mathrm{r}}^{*}=\left[\frac{R_{\mathrm{b}}^{*}\left(t^{*}=t_{\text {core }}^{*}\right)}{\ell_{\mathrm{ED}} t_{\text {core }}^{*}}-\frac{3-s}{n-3} \frac{v_{\mathrm{b}}^{*}\left(t^{*}=t_{\text {core }}^{*}\right)}{\ell_{\mathrm{ED}}} \ln \frac{t^{*}}{t_{\text {core }}^{*}}\right] t^{*}$, 

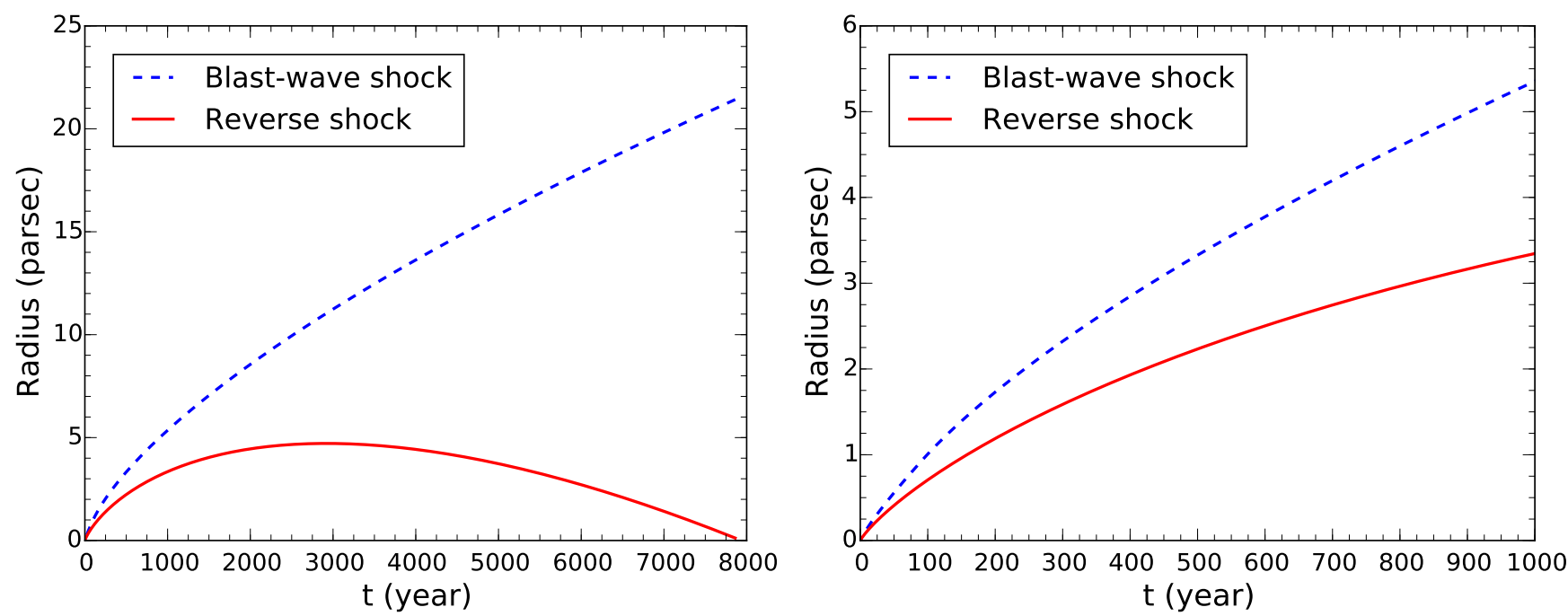

Fig. 3. Left: blast-wave and reverse shock radii as a function of the time elapsed since explosion. Right: zoom of left panel for $t \leq 1000 \mathrm{yr}$.

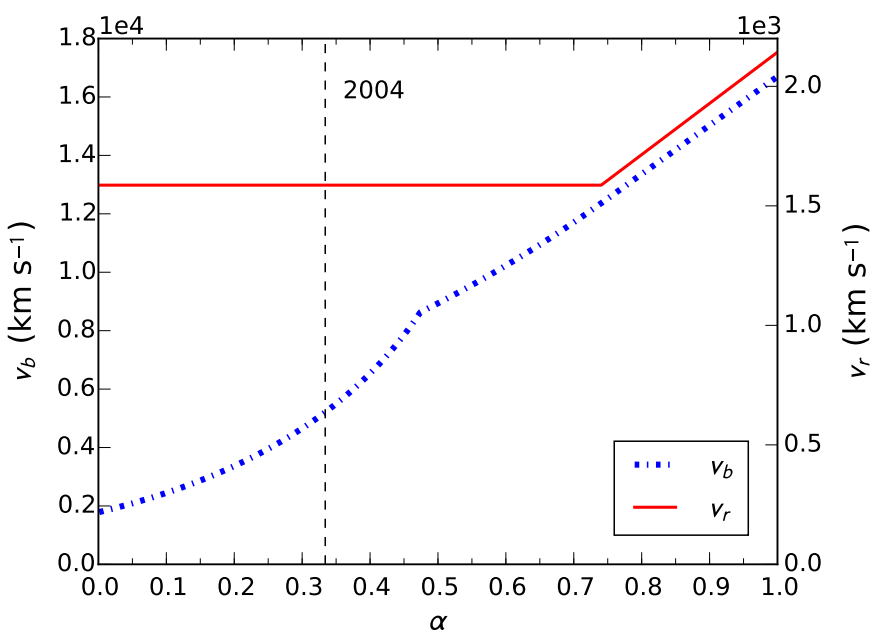

Fig. 4. Blast-wave and reverse shock velocities ( $v_{\mathrm{b}}$ and $v_{\mathrm{r}}$, respectively) as a function of the parameter $\alpha$. The reverse shock velocity (right $y$-axis, in units of $10^{3} \mathrm{~km} \mathrm{~s}^{-1}$ ) is calculated in the frame of the unshocked ejecta ahead of it. We assume that after the reverse shock has entered the ejecta core $(\alpha<0.75)$, its velocity remains constant and equal to the value for $t^{*}=t_{\text {core }}^{*}$ (Eq. (34), see text). For each value of $\alpha$, the curve for $v_{\mathrm{b}}$ (left $y$-axis, in units of $10^{4} \mathrm{~km} \mathrm{~s}^{-1}$ ) provides the velocity of the blast-wave shock at the moment when the reverse shock hits a layer $\alpha$ of the ejecta. Vertical line as in Fig. 1.

and the reverse shock velocity is given by

$v_{\mathrm{r}}^{*}=\frac{3-s}{n-3} \frac{v_{\mathrm{b}}^{*}\left(t^{*}=t_{\mathrm{core}}^{*}\right)}{\ell_{\mathrm{ED}}}$

We assume that inside the core the reverse shock velocity remains constant and equal to the value for $t^{*}=t_{\text {core }}^{*}$.

The radius of the blast-wave shock (Eqs. (24) and (26)) and of the reverse shock (Eqs. (31) and (33)) as a function of the time elapsed since the progenitor of Cas A exploded as a supernova are shown in Fig. 3. The velocity of the blast-wave shock (Eqs. (25) and (27)) and of the reverse shock (Eqs. (32) and (34)) as a function of the parameter $\alpha$ are shown in Fig. 4.

\section{Application to Cas A}

\subsection{Ejecta geometry and physical properties}

The Cas A supernova remnant is the result of the explosion of a Type IIb supernova (Krause et al. 2008) with a progenitor mass estimated between 15 and $25 M_{\odot}$ (Young et al. 2006; Vink et al. 1996). The remnant is located at a distance of $3.4 \mathrm{kpc}$ (Reed et al. 1995) and its age was 333 yr in 2004 (date of the Chandra observations which have been used to determine some of the parameters of Cas A used in this work). From our calculations (Sect. 2.3) we obtain the following values for the radius and velocity of the forward and reverse shock of Cas A in 2004: $R_{\mathrm{b}}=2.5 \mathrm{pc}, v_{\mathrm{b}}=5226 \mathrm{~km} \mathrm{~s}^{-1}, R_{\mathrm{r}}=1.71 \mathrm{pc}$ and $v_{\mathrm{r}}=$ $1586 \mathrm{~km} \mathrm{~s}^{-1}$. Our numbers are consistent with the values derived from observations: $R_{\mathrm{b}}=2.32-2.72 \mathrm{pc}, v_{\mathrm{b}}=4000-6000 \mathrm{~km} \mathrm{~s}^{-1}$, $R_{\mathrm{r}}=1.52-2.01 \mathrm{pc}, v_{\mathrm{r}}=1000-2000 \mathrm{~km} \mathrm{~s}^{-1}$ (see Table 1 for the corresponding references).

We obtained this nice match adopting the ejecta mass $M_{\mathrm{ej}}=$ $2 M_{\odot}$ (consistent with the value of $2.2 M_{\odot}$ inferred by Willingale et al. 2002, 2003), and the explosion energy $E=2.2 \times 10^{51} \mathrm{erg}$, in agreement with the amount of energy expected from a corecollapse SN (Laming \& Hwang 2003), and the circumstellar (preshock) density $n_{0}=2.07 \mathrm{H}$ atom $\mathrm{cm}^{-3}$, which is compatible with the value of $1.99 \mathrm{~cm}^{-3}$ from Willingale et al. (2003).

The density distribution of the supernova ejecta is described in a more realistic and observationally motivated way by a series of overdense clouds embedded into a smooth and tenuous medium (e.g. Peimbert \& van den Bergh 1971; Kamper \& van den Bergh 1976; Chevalier \& Kirshner 1978, 1979; Fesen et al. 2001; Rho et al. 2009, 2012; Wallström et al. 2013). For the ejecta clouds, we assume a pre-shock density $n_{\text {cloud }}=$ $100 \mathrm{~cm}^{-3}$ with a density contrast with respect to the smooth component $\chi=n_{\text {cloud }} / n_{\text {smooth }}=100$. These values allow to reproduce the optical spectra of the fast moving knots (FMKs) in Cas A in a fairly accurate way (Sutherland \& Dopita 1995). For the smooth ejecta, therefore, we obtain the pre-shock density $n_{\text {smooth }}=n_{\text {cloud }} / \chi=1.0 \mathrm{~cm}^{-3}$, which is consistent with the ambient density of $0.1-10 \mathrm{~cm}^{-3}$ (average $\sim 0.25 \mathrm{~cm}^{-3}$ ) estimated for the X-ray emitting gas on the bases of ram pressure arguments (Morse et al. 2004). This value for the smooth ejecta density should be considered as indicative. To evaluate the effect 
Table 1. Physical properties of Cas A.

\begin{tabular}{lllll}
\hline \hline \multicolumn{1}{c}{ Parameter } & Symbol (units) & $\begin{array}{c}\text { Calculated// } \\
\text { adopted } \\
\text { value }\end{array}$ & $\begin{array}{c}\text { Observed/ } \\
\text { estimated } \\
\text { value }\end{array}$ & \multicolumn{1}{c}{ References } \\
\hline Progenitor mass & $\left(M_{\odot}\right)$ & $19^{a}$ & $15-25$ & 1,2 \\
Distance & $(\mathrm{kpc})$ & $3.4^{a}$ & 3.4 & 3 \\
Age (2004) & $(\mathrm{yr})$ & $333^{a}$ & 333 & 4 based on 5 \\
Explosion energy & $E\left(10^{51} \mathrm{erg}\right)$ & $2.2^{b}$ & 2.0 & 6 \\
Ejecta mass & $M_{\mathrm{ej}}\left(M_{\odot}\right)$ & $2.0^{b}$ & 2.2 & 7,8 \\
Forward shock radius & $R_{\mathrm{b}}(\mathrm{pc})$ & $2.50^{c, *}$ & $2.32-2.72$ & $9,10,11$ \\
Reverse shock radius & $R_{\mathrm{r}}(\mathrm{pc})$ & $1.71^{c, *}$ & $1.52-2.01$ & $9,10,11,12$ \\
Forward shock velocity & $v_{\mathrm{b}}\left(\mathrm{km} \mathrm{s}^{-1}\right)$ & $5226^{c, *}$ & $4000-6000$ & 13 \\
Reverse shock velocity & $v_{\mathrm{r}}\left(\mathrm{km} \mathrm{s}^{-1}\right)$ & $1586^{c, *}$ & $1000-2000$ & 6,12 \\
Circumstellar density (pre-shock) & $n_{0}\left(\mathrm{~cm}^{-3}\right)$ & $2.07^{b}$ & 1.99 & 8 \\
Density contrast clumps/smooth ejecta & $\chi$ & $100^{a}$ & $\approx 100$ & 14 \\
Ejecta clumps density (pre-shock) & $n_{\text {cloud }}\left(\mathrm{cm}^{-3}\right)$ & $100^{a}$ & $100-300$ & 14,15 \\
Smooth ejecta density (pre-shock) & $\left.n_{\text {smooth }\left(\mathrm{cm}^{-3}\right)}\right)$ & $1.0^{c}$ & $0.1-10$ & 12 \\
Ejecta clumps diameter & $2 R_{\text {cloud }}\left(\mathrm{cm}^{c}\right)$ & $1.5 \times 10^{16 a}$ & $(1-5) \times 10^{16}$ & 16 \\
\hline
\end{tabular}

Notes. ${ }^{(a)}$ Adopted. ${ }^{(b)}$ Allows a good match between measured and calculated values. ${ }^{(c)}$ Calculated - this work. ${ }^{(*)}$ Value in 2004.

References. [1] Young et al. (2006), [2] Vink et al. (1996), [3] Reed et al. (1995), [4] Hwang \& Laming (2012), [5] Thorstensen et al. (2001), [6] Laming \& Hwang (2003), [7] Willingale et al. (2002), [8] Willingale et al. (2003), [9] Hwang \& Laming (2012), [10] Helder \& Vink (2008), [11] Gotthelf et al. (2001), [12] Morse et al. (2004), [13] DeLaney \& Rudnick (2003), [14] Sutherland \& Dopita (1995), [15] Docenko \& Sunyaev (2010), [16] Fesen et al. (2011).

of dust sputtering we use the density structure shown in Fig. 2, which has been calculated with our model for the evolution of the Cas A ejecta. Our model predicts for 2004 a number density of $0.23 \mathrm{~cm}^{-3}$, which is very close to the average value estimated by Morse et al. (2004).

The most recent determinations of the amounts of dust produced in Cas A were carried out by Barlow et al. (2010) and Arendt et al. (2014). The warm dust emission at 5-35 $\mu \mathrm{m}$ observed by Spitzer is mostly generated by $\sim 0.04 M_{\odot}$ of mainly silicate dust. Most of the dust mass is associated with a colder dust component that dominates the broadband emission at wavelengths $\gtrsim 60 \mu \mathrm{m}$ and is associated with the Si II] $34.82 \mu \mathrm{m}$ line emission. Because of the lack of any distinguishing dust emission features at these wavelengths, its composition could not be uniquely determined and its mass was estimated to be $<0.1 M_{\odot}$. This cold dust component has probably not yet encountered the reverse shock. Its distribution is very different from the warm dust that is spatially coincident with reverse shock features. We assume that the dust, initially present solely in the clumps, does not make the clumps affect the dynamics of the reverse shock.

The ejecta clouds become visible due to reverse shock heating and they constitute the bright main shell of the remnant. Optical maps show that the diameter of the ejecta clumps, $D=$ $2 R_{\text {cloud }}$, is in the range $0.2-1.0^{\prime \prime}$ (Fesen et al. 2011), which corresponds to $(1-5) \times 10^{16} \mathrm{~cm}$ at a distance of $3.4 \mathrm{kpc}^{1}$. These clumps are bigger than the knots located outside the main shell of the remnant, at or ahead of the forward shock front (Fesen et al. 2006; Hammell \& Fesen 2008), whose size is typically lower than $\sim 10^{15} \mathrm{~cm}$ (Fesen et al. 2011). Kamper \& van den Bergh (1976) and van den Bergh \& Kamper (1985) found that optical clumps brighten up and gradually fade with time, and the number of clouds visible at any time is described well by an exponential decay that has an $e$-folding time of around $25 \mathrm{yr}$

\footnotetext{
1 In Docenko \& Sunyaev (2010) there is a misprint in the linear size of the clumps, which is erroneously reported as $(3-15) \times 10^{16} \mathrm{~cm}$ instead of $(1-5) \times 10^{16} \mathrm{~cm}$ (at a distance of $3.4 \mathrm{kpc}$ ).
}

(Kamper \& van den Bergh 1976). We adopt as a representative cloud size the intermediate value $2 R_{\text {cloud }}=1.5 \times 10^{16} \mathrm{~cm} \simeq$ $0.005 \mathrm{pc}$, which provides a cloud lifetime (see below) that is consistent with the aforementioned determination from Kamper \& van den Bergh (1976).

When the reverse shock encounters an ejecta cloud, it generates a cloud shock which propagates into the cloud with velocity $v_{\text {cloud }}=v_{\mathrm{r}} / \sqrt{\chi}$. The clumps heated up by the shock become visible before fading gradually away. The timescale for this process is correlated to the time required by the cloud shock to reach the opposite side of the clump. This latter time is given by twice the cloud crushing time, $t_{\mathrm{cc}} \equiv R_{\text {cloud }} / v_{\text {cloud }}$ (Klein et al. 1994), which in addition provides an indication of the dynamical timescale for shock-induced cloud fragmentation.

From our calculated value $v_{\mathrm{r}} \sim 1600 \mathrm{~km} \mathrm{~s}^{-1}$, we obtain $v_{\text {cloud }}=160 \mathrm{~km} \mathrm{~s}^{-1}$, which gives $2 t_{\mathrm{cc}}=2 R_{\text {cloud }} / v_{\text {cloud }}=30 \mathrm{yr}$, in agreement with the lifetime of the Cas A FMKs as deduced from optical observations (Kamper \& van den Bergh 1976, see above). The physical properties of Cas A are summarized in Table 1. Figure 5 shows a schematic illustration of the propagation of the reverse shock into the smooth ejecta and through an ejecta clump.

The ejecta clouds contain $80 \%-90 \%$ of oxygen, with a small contribution from $\mathrm{Ne}, \mathrm{Si}, \mathrm{S}, \mathrm{Ar}$, and negligible abundances of hydrogen and helium (Docenko \& Sunyaev 2010). Optical observations have revealed some knots made of almost pure oxygen, dubbed [O III] filaments (Chevalier \& Kirshner 1979). This justifies our simplifying assumption of ejecta clouds with a composition of $100 \%$ oxygen.

We assume that all the dust resides in the oxygen rich clumps and that it is composed of silicates $\left(\mathrm{MgSiO}_{3}\right)$ and amorphous carbon. Because the initial dust grain size distribution of the newly formed dust is not determined well, as a test case we adopt the classical MRN power-law expression (Mathis et al. 1977): $g(a) \sim a^{-3.5}$ and $a_{\min }<a<a_{\max }$, with $a_{\min }=50 \AA$ and $a_{\max }=2500 \AA$. The quantity $g(a)$ represents the number of grains with radius $a$ in the interval $\mathrm{d} a$. 


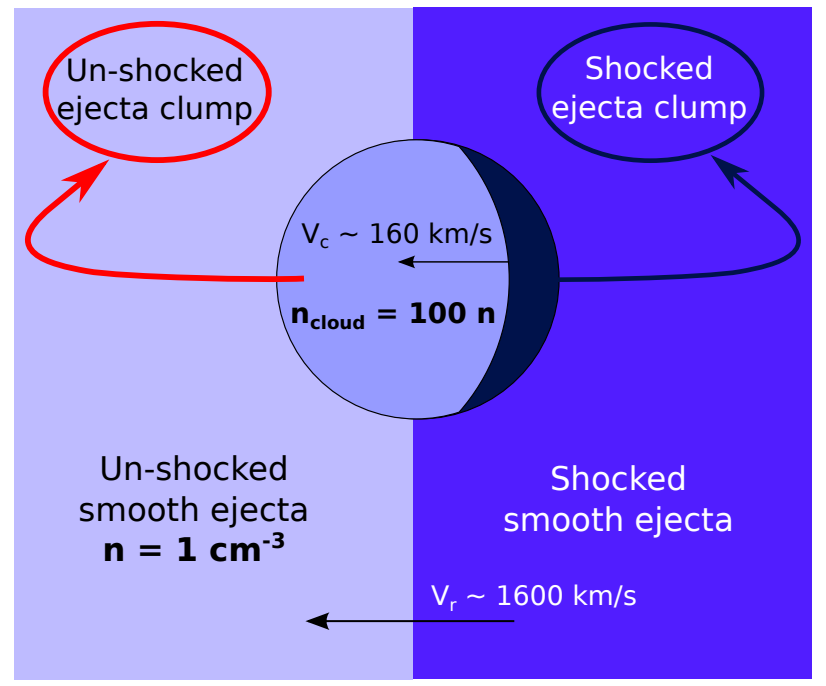

Fig. 5. Schematic illustration of the propagation of the reverse shock (velocity $v_{\mathrm{r}} \sim 1600 \mathrm{~km} \mathrm{~s}^{-1}$ ) into the smooth ejecta and through an ejecta clump. The reverse shock hitting the clump generates a cloud shock that propagates with velocity $v_{\mathrm{c}} \equiv v_{\text {cloud }}=v_{\mathrm{r}} / \sqrt{\chi}$, where we assume $\chi=n_{\text {cloud }} / n_{\text {smooth }}=100$ and $n_{\text {cloud }}=100 \mathrm{~cm}^{-3}$, which leads to $n_{\text {smooth }} \equiv$ $n=1 \mathrm{~cm}^{-3}$. Figure adapted from Micelotta (2013).

Table 2. Elemental masses in the smooth ejecta of Cas A.

\begin{tabular}{llc}
\hline \hline Element & $M_{i} / M_{\mathrm{O}}{ }^{a}$ & $M_{i} / M_{\mathrm{H}}{ }^{b}$ \\
\hline $\mathrm{O}$ & 1.00 & $8.59 \times 10^{-3}$ \\
$\mathrm{Ne}$ & 0.015 & $1.28 \times 10^{-4}$ \\
$\mathrm{Mg}$ & 0.0039 & $3.35 \times 10^{-5}$ \\
$\mathrm{Si}$ & 0.021 & $1.80 \times 10^{-4}$ \\
$\mathrm{~S}$ & 0.011 & $9.45 \times 10^{-5}$ \\
$\mathrm{Ar}$ & 0.0056 & $4.81 \times 10^{-5}$ \\
$\mathrm{Fe}$ & $0.057^{c}$ & $4.90 \times 10^{-4}$ \\
\hline
\end{tabular}

Notes. ${ }^{(a)}$ Derived from X-rays observations (Hwang \& Laming 2012, Table 2). ${ }^{(b)}$ Calculated from $M_{i} / M_{\mathrm{O}}$ - see text for details. ${ }^{\left({ }^{c}\right)}$ Sum of two iron components: 0.041+0.016 (Hwang \& Laming 2012).

For the smooth (X-ray emitting) ejecta, we assume a composition of $\mathrm{O}, \mathrm{Ne}, \mathrm{Mg}, \mathrm{Si}, \mathrm{S}, \mathrm{Ar}$, and $\mathrm{Fe}$ as determined by Hwang \& Laming (2012) and reported in their Table 2. This work provides the elemental masses with respect to oxygen, $M_{i} / M_{\mathrm{O}}$. To convert them to $M_{i} / M_{\mathrm{H}}$, elemental masses with respect to hydrogen, we use the equation $M_{i} / M_{\mathrm{H}}=$ $\left(M_{i} / M_{\mathrm{O}}\right)\left(M_{\mathrm{O}} / M_{\mathrm{H}}\right)$, where the protosolar $M_{\mathrm{O}} / M_{\mathrm{H}}=8.59 \times 10^{-3}$ is given by Table 1.4 in Draine (2011) and based on Asplund et al. (2009). The values of all masses are reported in Table 2.

\subsection{Temperature evolution of the ejecta}

When a shock propagates through a gas, it heats the gas located right beyond the shock front up to a temperature $T$ given by the following expression:

$T=\frac{3}{16} \frac{\mu}{k_{\mathrm{B}}} v_{\mathrm{s}}^{2}$,

where $\mu$ is the mean molecular mass per particle and $v_{\mathrm{s}}$ is the velocity of the shock. For a mixture of neutral elements, $\mu$ is written as

$\mu_{\text {neutral }}=\sum_{i} \frac{M_{i}}{M_{\mathrm{H}}} \times \mathrm{amu}$, where amu is the atomic mass unit. For a mixture of ionized elements in different ionization states $j$, we have

$\mu_{\text {ionized }}=\frac{\mu_{\text {neutral }}}{\text { ion }}$

The quantity ion represents the total number of particles (nuclei plus electrons) per hydrogen atom and can be calculated from the following relation:

ion $=\sum_{i} \frac{N_{i}}{N_{\mathrm{H}}}\left[\sum_{j} f_{i j}\left(1+N_{\mathrm{e}}^{i j}\right)\right]$.

The index $i$ runs over the elemental species and the index $j$ runs over the ionization states. For each species $i$, the quantity $N_{i} / N_{\mathrm{H}}=\left(M_{i} / M_{\mathrm{H}}\right) /\left(\left\langle m_{i}\right\rangle / \mathrm{amu}\right)$ represents the number of atoms per $\mathrm{H}$ atom, where $\left\langle m_{i}\right\rangle / \mathrm{amu}$ is the mean mass in atomic units. The number of electrons ejected per atom $i$ in the ionization state $j$ is given by $N_{\mathrm{e}}^{i j}$ and the fraction of such atoms by $f_{i j}$.

In the ejecta clouds, the rise of temperature is due to the cloud shock, which has velocity $v_{\text {cloud }}=160 \mathrm{~km} \mathrm{~s}^{-1}$. As mentioned above we assume a composition of pure oxygen, and an ionization state of +2 . This implies that ion $=1+2=3$ and $\mu=5.34 \times$ amu $=8.9 \times 10^{-24} \mathrm{~g}$. Using the above equations, we obtain the cloud temperature $T_{\text {cloud }} \sim 3 \times 10^{6} \mathrm{~K}$. This is consistent with the results from Sutherland \& Dopita (1995) and Borkowski \& Shull (1990), who obtained temperatures of $2.75 \times 10^{6} \mathrm{~K}$ and $3.46 \times 10^{6} \mathrm{~K}$ for cloud shock velocities of $150 \mathrm{~km} \mathrm{~s}^{-1}$ and $170 \mathrm{~km} \mathrm{~s}^{-1}$, respectively.

Equation (35) assumes equilibration between the ion and electron temperatures. The work of Itoh (1981) on the emission from oxygen-rich supernova ejecta (the case of Cas A) shows that in the immediate post-shock region the electron temperature, $T_{\mathrm{e}}$, is much lower than the ion temperature, $T_{\text {ion }}$. Figure 2 however indicates that $T_{\mathrm{e}}$ and $T_{\text {ion }}$ equilibrate over a scale length of $\sim 4 \times 10^{13} \mathrm{~cm}$, which corresponds to only $\sim 0.27 \%$ of the diameter of our clumps. This is confirmed by recent shock calculations performed for cases comparable to those studied here, which show that both the equilibration and the cooling length scales are very short, $\sim 2 \times 10^{13} \mathrm{~cm}$ at most even for the case of $T_{\mathrm{e}} / T_{\text {ion }}=0.01$ at the shock front (J. Raymond, priv. comm.). Such results imply that temperature equilibration occurs quickly; these results are in agreement with van Adelsberg et al. (2008) and Ghavamian et al. (2007), who both find that full equilibration occurs in non-radiative shocks (not necessarily strongly cooling) for shock velocities below $\sim 400 \mathrm{~km} \mathrm{~s}^{-1}$. The assumption of temperature equilibration appears therefore justified in the present case.

The shock propagating into the smooth ejecta is the reverse shock with velocity $v_{\mathrm{r}}=1600 \mathrm{~km} \mathrm{~s}^{-1}$. To calculate the resulting temperature of the shocked smooth ejecta, we would need the ionization state of the pre-shock gas, which is pre-ionized by the incoming reverse shock. Unfortunately, there are no determinations of such an ionization state. To make an estimate, we use the results from the photo-ionization and shock modelling code Mappings III (Allen et al. 2008). For the elemental composition given by Hwang \& Laming (2012), we adopt for each element the ionization state calculated by Mappings III for a gas of solar abundances, with number density $n=1 \mathrm{~cm}^{-3}$, pre-ionized by a shock with velocity of $1000 \mathrm{~km} \mathrm{~s}^{-1}$ (Model M). We adopt a transverse magnetic field $B=1 \mu \mathrm{G}$, to be consistent with the model of Sutherland \& Dopita (1995) who assumed this value for the ejecta clumps; we make the assumption that the pre-shock magnetic field is the same in both the clumps and smooth ejecta. Such 
a magnetic field does not affect the temperature or dynamics, but it would imply betatron acceleration of the charged grains, which would affect the cloud shock. In a following study, we will investigate the modifications of the velocity profiles of the grains induced by betatron acceleration and their impact on the dust sputtering process.

The set of parameters described above is the closest match to Cas A among those available from the code. The resulting ionization states are the following: $\mathrm{O}^{6+}, \mathrm{Ne}^{5+}, \mathrm{Mg}^{7+}, \mathrm{Si}^{8+}$, $\mathrm{S}^{8+}, \mathrm{Ar}^{6+}, \mathrm{Fe}^{7+}$. Because the maximum shock velocity considered by Mappings III is lower than the velocity of the reverse shock in Cas A $\left(v_{\mathrm{r}}=1600 \mathrm{~km} \mathrm{~s}^{-1}\right)$, we expect that the resulting ionization states are underestimated. With these parameters, the temperature of the shocked smooth ejecta from Eq. (35) is $T_{\text {smooth }}=1.38 \times 10^{8} \mathrm{~K}$. Even if we consider the extreme (and unrealistic) case of complete pre-ionization of the pre-shock gas, the temperature is only reduced to $1.02 \times 10^{8} \mathrm{~K}$.

It should be noted that in the smooth ejecta the hypothesis of equal ion and electron temperatures may not be fully justified. Yamaguchi et al. (2014) show that in the reverse shock of Tycho there is some electron heating via ion-electron Coulomb collisions, but that it is weak. The results from van Adelsberg et al. (2008) would indicate that the electron-to-ion temperature ratio, $\beta \equiv T_{\mathrm{e}} / T_{\text {ion }}$, has a value of $\sim 3-8 \%$ for a shock speed of $1600 \mathrm{~km} \mathrm{~s}^{-1}$. In Cas $\mathrm{A}$ the electron temperature derived from X-ray observations is $\sim 1.7 \times 10^{7} \mathrm{~K}\left(k_{\mathrm{B}} T=1-2 \mathrm{eV}\right.$; Hwang $\&$ Laming 2009). This number combined with the values of $\beta$ reported above gives $T_{\text {ion }}=(2-5.7) \times 10^{8} \mathrm{~K}$, which is higher than the temperature derived from Eq. (35). The implications of this situation will be evaluated in a follow-up work, while for the present study we maintain the hypothesis of ion-electron temperature equilibration in the smooth ejecta.

The temperature of the gas in which the dust is embedded determines the type and level of processing of the grains. It is therefore important to evaluate the cooling of both the cloud and smooth ejecta. To determine the cooling time as a function of temperature, $\tau_{\text {cool }}(T)$, we adopt the formalism from Sutherland \& Dopita (1993). For a gas composed of only one elemental species in a single ionization state, this gives

$$
\begin{aligned}
\tau_{\text {cool }}(T) & =\frac{\mathcal{U}}{n_{\mathrm{e}} n_{i} \Lambda(T)}=\frac{(3 / 2) \mathcal{N} k_{\mathrm{B}} T}{n_{\mathrm{e}} n_{i} \Lambda(T)} \\
& =\frac{3}{2} \frac{\left(n_{\mathrm{e}}+n_{i}\right) k_{\mathrm{B}} T}{n_{\mathrm{e}} n_{i} \Lambda(T)} .
\end{aligned}
$$

In this set of equations, $\mathcal{U}$ is the internal energy, $\Lambda(T)$ the cooling function and $\mathcal{N}=n_{\mathrm{e}}+n_{i}$, where $n_{\mathrm{e}}$ and $n_{i}$ are the number density of electrons and ions, respectively. Because $n_{\mathrm{e}}=A n_{i}$, where $A$ is the number of electrons ejected from each atom (i.e. its ionization state), Eq. (40) can be rewritten in a more general form as

$\tau_{\text {cool }}(T)=\frac{3}{2} \frac{A+1}{A} \frac{k_{\mathrm{B}} T}{n_{i} \Lambda(T)}$.

We have assumed a composition of pure oxygen for the ejecta clumps, which implies that $n_{i}=n_{\mathrm{O}}=n_{\text {cloud }}$. For the cooling function of the shocked ejecta clouds, we use the values computed by Sutherland \& Dopita (1995, see their Fig. 2) for $v_{\text {cloud }}=150 \mathrm{~km} \mathrm{~s}^{-1}$, which is very close to our own value of $160 \mathrm{~km} \mathrm{~s}^{-1}$. To our knowledge, this study provides the only available estimate of the cooling function of an oxygen-rich shocked gas. To determine $n_{\mathrm{e}}$, we would need the oxygen ionization structure for a $160 \mathrm{~km} \mathrm{~s}^{-1}$ cloud shock, which is not available. Instead, we use the ionization structure of the $200 \mathrm{~km} \mathrm{~s}^{-1}$ shock model

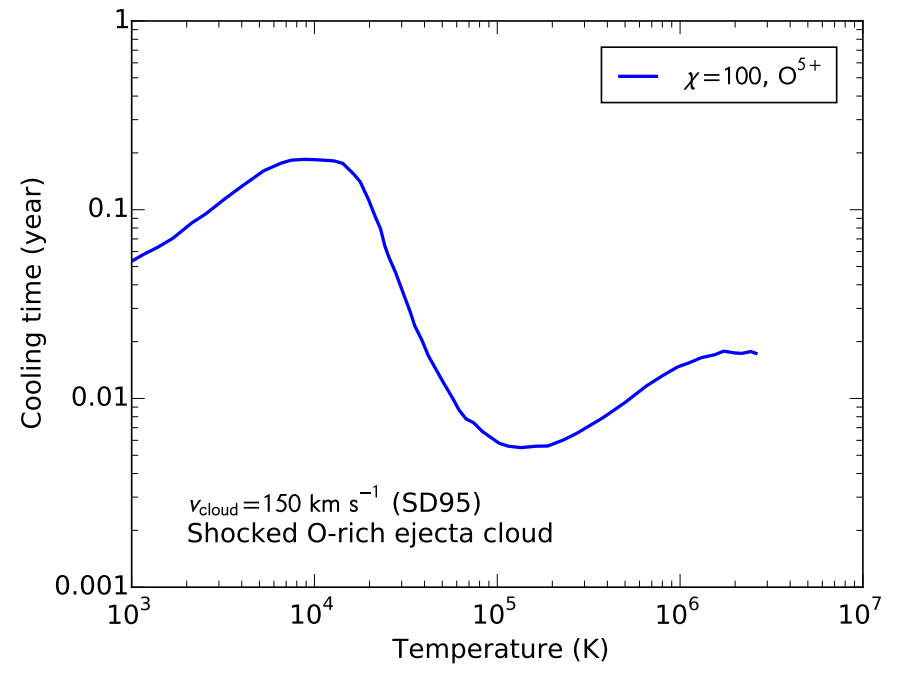

Fig. 6. Cooling time as a function of temperature for a shocked O-rich ejecta cloud in Cas A, calculated from Eq. (42).

from Sutherland \& Dopita (1995) shown in their Fig. 3 (lower panel), which gives $\mathrm{O}^{5+}$ as the dominant ionization state. This results in $A=5, n_{\mathrm{e}}=5 n_{\mathrm{i}}$ and $\mathcal{N}=5 n_{i}+n_{i}=6 n_{i}$. Docenko $\&$ Sunyaev (2010) performed their own calculation of the postshock oxygen ion distribution for a $200 \mathrm{~km} \mathrm{~s}^{-1}$ cloud shock obtaining results similar to Sutherland \& Dopita (1995), but only if the spectroscopic symbols in the original figure are increased by unity. Docenko \& Sunyaev (2010) assumed a misprint. In this case, the dominant ionization state would be $\mathrm{O}^{6+}$, which leads to $A=6, n_{\mathrm{e}}=6 n_{i}$, and $\mathcal{N}=7 n_{i}$.

For our shocked ejecta cloud, the number density to include in Eq. (41) is that in the immediate post-shock gas, which is increased by a factor of 4 with respect to the unshocked gas. As a result, for the number density of the shocked cloud we have $n_{i}^{\text {sc }}=4 n_{i}$. The cooling time of a shocked ejecta cloud is then given by Eq. (41) modified as follows

$$
\tau_{\text {cool }}^{\mathrm{sc}}(T)=\frac{3}{2} \frac{A+1}{A} \frac{k_{\mathrm{B}} T}{4 n_{i} \Lambda(T)} .
$$

The cooling time $\tau_{\text {cool }}^{\text {sc }}(T)$ from Eq. (42) is shown in Fig. 6 assuming $\mathrm{O}^{5+}$ as the dominant ionization state. If in the plot showing the oxygen ionization structure for the $200 \mathrm{~km} \mathrm{~s}^{-1}$ cloud shock model (Fig. 3, lower panel in Sutherland \& Dopita 1995) there is indeed the misprint indicated by Docenko \& Sunyaev (2010), the dominant ionization state for oxygen would be $\mathrm{O}^{6+}$. In this case, the cooling time for the shocked cloud is given again by Eq. (42) with the same cooling function $\Lambda(T)$, but adopting $A=6$. The curves for $\mathrm{O}^{5+}$ and $\mathrm{O}^{6+}$ are indistinguishable (the ratio between the two is $3 \%$ ), so we consider negligible the variation induced by an eventual misprint and follow Sutherland \& Dopita (1995) adopting $\mathrm{O}^{5+}$ as the dominant ionization state produced by a $200 \mathrm{~km} \mathrm{~s}^{-1}$ shock propagating into an oxygen-rich cloud. To determine the temporal evolution of the gas temperature in the shocked cloud, we have to solve the following differential equation:

$$
\frac{\mathrm{d} T}{\mathrm{~d} t}=-\frac{T}{\tau_{\mathrm{cool}}^{\mathrm{sc}}(T)},
$$

with $\tau_{\text {cool }}^{\mathrm{sc}}(T)$ from Eq. (42). The solution of the equation is shown in Fig. 7. Because of the extreme metallicity, it takes only six months for the temperature of the ejecta in the clouds to go 


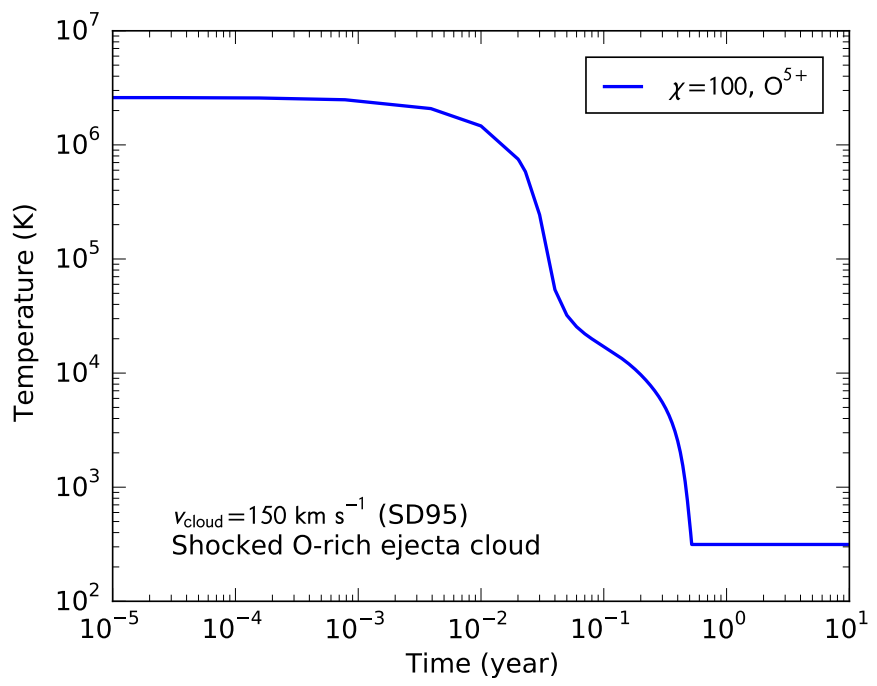

Fig. 7. Temperature evolution as a function of time for a shocked O-rich ejecta cloud in Cas A, calculated from Eq. (43) using the cooling time from Eq. (42).

from $\sim 3 \times 10^{6} \mathrm{~K}$ to $300 \mathrm{~K}$. While the cloud shock progresses through the clump, the layer beyond the shock front with a temperature close to $\sim 10^{5} \mathrm{~K}$, i. e. high enough for thermal sputtering to be effective, has a thickness of $\sim 2 \times 10^{14} \mathrm{~cm}$; this layer is only $1.4 \%$ of the diameter of the clump. The small amount of dust in such a thin layer allows us to neglect thermal sputtering and to assume that, while residing in the ejecta clumps, the dust is affected only by inertial (kinetic) sputtering (see Sect. 4).

To estimate the temperature evolution of the shocked smooth ejecta, we used Mappings III with appropriate modifications of the input parameters, to obtain the closest match with the observed properties of Cas A. The calculations are performed under non-equilibrium ionization (NEI) conditions. We adopted the elemental composition determined by Hwang \& Laming (2012) for the smooth ejecta in Cas A and run the code for a shock with the velocity of the reverse shock, $v_{\mathrm{r}}=1600 \mathrm{~km} \mathrm{~s}^{-1}$. This value is above the nominal limit of the code $\left(1000 \mathrm{~km} \mathrm{~s}^{-1}\right)$ and is expected to increase the pre-ionization state of the pre-shock gas. However, we have shown early on in this section that even a complete pre-ionization does not significantly modify the resulting post-shock temperature. We consider therefore that the calculation could provide a reasonable estimate of the temperature evolution for the faster shock as well. The result is shown in Fig. 8 and is consistent with our previous calculation. With the same ejecta composition but a shock velocity of $1000 \mathrm{~km} \mathrm{~s}^{-1}$, we obtain a post-shock temperature of the order of $10^{7} \mathrm{~K}$, which is consistent with the value of the electron temperature derived from X-ray observations (Hwang \& Laming 2009). The same calculations performed using the updated code Mappings $\mathrm{IV}^{2}$, give very similar results. As can be seen in Fig. 8, in both cases, after a phase of rapid cooling $(\sim 10 \mathrm{yr})$ the temperature remains fairly constant at $\sim 10^{8} \mathrm{~K}$ and $\sim 10^{7} \mathrm{~K}$ for more than $10^{4} \mathrm{yr}$ before dropping abruptly.

\section{Grain destruction by the reverse shock: Kinetic sputtering}

The rate at which a dust grain is sputtered as a result of its relative motion through a gas, a process also known as kinetic

\footnotetext{
2 http://miocene.anu.edu.au/miv/
}

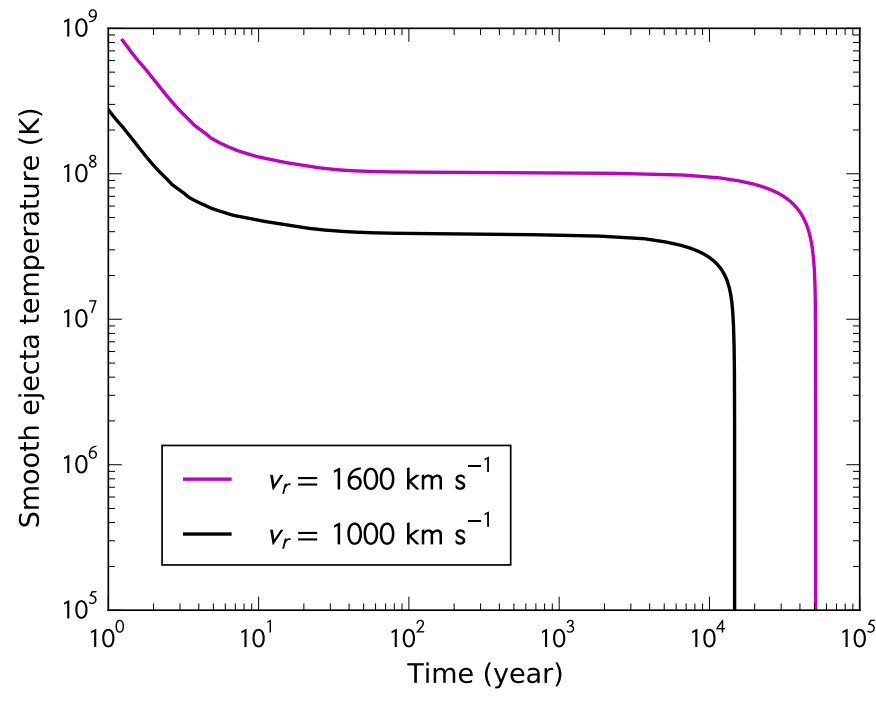

Fig. 8. Temperature evolution as a function of time in the shocked smooth ejecta, calculated for two shock velocities under nonequilibrium ionization (NEI) conditions, using the Mappings III code.

or inertial sputtering, is given by the sum over colliding gas species $i$ (e.g. Dwek \& Arendt 1992), i.e.

$\frac{\mathrm{d} m_{\mathrm{gr}}}{\mathrm{d} t}=2 \pi a_{\mathrm{gr}}^{2} m_{\mathrm{sp}} \sum_{i} n_{i} v_{\mathrm{gr}} Y_{i}\left(E_{i}=m_{i} v_{\mathrm{gr}}^{2} / 2\right)$,

where $a_{\mathrm{gr}}$ is the grain radius; $m_{\mathrm{sp}}$ is the average mass of the atom/molecule sputtered from the grain, $v_{\mathrm{gr}}$ the grain velocity relative to the ambient gas, which is equivalent to the velocity of the incident projectile seen by a target grain considered stationary; $n_{i}$ is the number density of the different gas species; $m_{i}$ is their atomic mass; and $Y_{i}$, the sputtering yield, is the number of atoms/molecules ejected from the grain per incident projectile of composition $i$ (Tielens et al. 1994; Nozawa et al. 2006). The factor of 2 in Eq. (44) corrects the yield, which is measured for normally incident projectiles on a target material for a distribution of incident angles.

For the sputtering yield $Y_{i}$ we adopt the expression given by Eq. (11) in Nozawa et al. (2006). This is the same provided by Tielens et al. (1994) except for the different formula used for the function $\alpha_{i}$, which appears in the yield and allows for a better agreement with sputtering data (for details see Nozawa et al. 2006; Tielens et al. 1994, and references therein).

For simplicity, we assume that all the grains in the SN ejecta are made of silicates (in the form of $\mathrm{MgSiO}_{3}$ ) and amorphous carbon (see Sect. 3.1). For these two kinds of grains, we adopt the sputtering parameters summarized in Table 3 . The quantities $M$ and $Z$ are the atomic mass and atomic number of the target material, respectively, $U_{0}$ is the surface binding energy, defined as the minimum energy which is necessary to transfer into the target to remove an atom from the top surface layer. The constant $K$ enters in one of the terms of the expression for the sputtering yield $Y_{i}(E)$ and has been determined via a comparison with laboratory experiments. Following Tielens et al. (1994) and Nozawa et al. (2006), for $\mathrm{MgSiO}_{3}$ we adopt the sputtering parameters of $\mathrm{SiO}_{2}$, which can be considered a good representative of silicates and for which experimental sputtering data are available. The mass of the ejected species is given by the average value $m_{\mathrm{sp}}=23 \mathrm{amu}$. For carbonaceous grains, we assume a composition of pure carbon, thus there is no hydrogenation following 

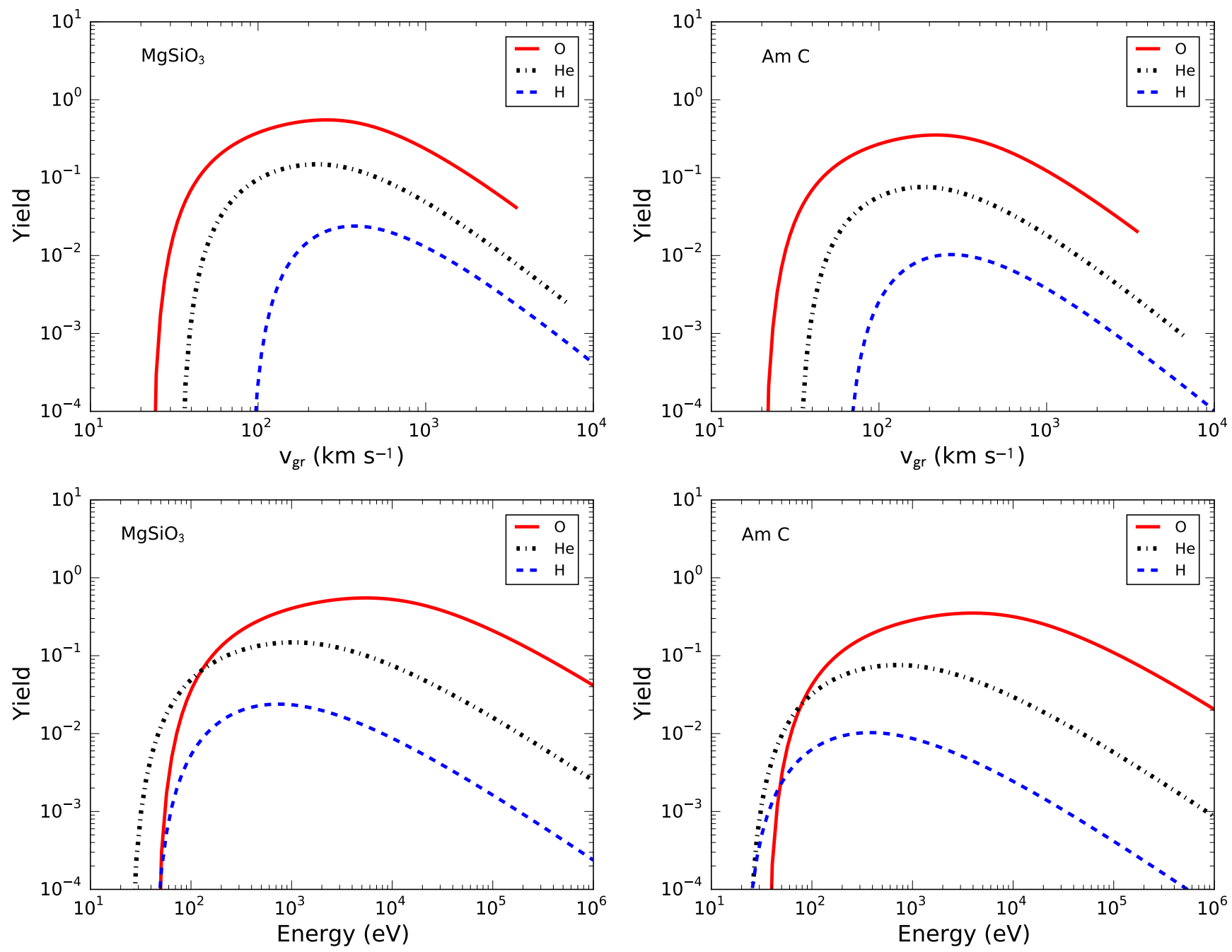

Fig. 9. Sputtering yields of silicate (left column) and amorphous carbon grains as a function of the relative grain-gas velocity (top row) and corresponding kinetic energy for the incident projectiles: oxygen, present in the ejecta clouds, helium, and hydrogen. The sputtering yields were calculated using Eq. (11) in Nozawa et al. (2006).

Table 3. Dust grain properties for sputtering calculations.

\begin{tabular}{ccccccc}
\hline \hline Material & $\begin{array}{c}\rho_{\mathrm{gr}} \\
\left(\mathrm{g} \mathrm{cm}^{-3}\right)\end{array}$ & $\begin{array}{c}U_{0} \\
(\mathrm{eV})\end{array}$ & $\begin{array}{c}M \\
(\mathrm{amu})\end{array}$ & $Z$ & $K$ & $\begin{array}{c}m_{\mathrm{sp}} \\
(\mathrm{amu})\end{array}$ \\
\hline $\mathrm{MgSiO}_{3}^{a}$ & 2.65 & 6.0 & 20 & 10 & 0.1 & 23 \\
$\mathrm{C}^{b}$ & 2.20 & 4.0 & 12 & 6 & 0.61 & 12 \\
\hline
\end{tabular}

Notes. Parameters $-\rho_{\mathrm{gr}}$ : mass density of the grains; $U_{0}$ : surface binding energy; $M$ and $Z$ : atomic mass and atomic number of the target material, respectively; $K$ : sputtering constant; and $m_{\text {sp }}$ : average mass of sputtered atoms.

References. ${ }^{(a)}$ Tielens et al. (1994); ${ }^{(b)}$ Nozawa et al. (2006) and references therein - Table 2 .

collisions because oxygen is the only projectile present in the clouds. As a consequence, the only sputtered atoms are carbon atoms, therefore, $m_{\mathrm{sp}}=12 \mathrm{amu}$.

Figure 9 presents the sputtering yield of amorphous carbon $(\mathrm{Am} \mathrm{C})$ and silicate $\left(\mathrm{MgSiO}_{3}\right)$ grains as a function of the velocity and kinetic energy of the incident projectiles, calculated using Eq. (11) in Nozawa et al. (2006).

Equation (44) assumes that the thermal velocities of the colliding atoms are negligible compared to the grain's velocity. The rate at which a grain slows down as a result of collisional drag forces is given by $\left(\mathrm{d} v_{\mathrm{gr}} / \mathrm{d} t\right)=F_{\mathrm{drag}} / m_{\mathrm{gr}}$. For grains moving through a cold gas $\left(2 k T \ll m_{\mathrm{gr}} v_{\mathrm{gr}}^{2}\right)$, the plasma correction to the drag force (Baines et al. 1965, Sect. 6) can be neglected and $F_{\text {drag }}$ is expressed as

$F_{\mathrm{drag}}=\pi a_{\mathrm{gr}}^{2} v_{\mathrm{gr}}^{2} \sum_{i} n_{i} m_{i}$

so that

$\frac{\mathrm{d} v_{\mathrm{gr}}}{\mathrm{d} t}=\frac{3}{4} \frac{v_{\mathrm{gr}}^{2}}{\rho_{\mathrm{gr}} a_{\mathrm{gr}}} \gamma \sum_{i} n_{i} m_{i}$,

where $\rho_{\mathrm{gr}}$ is the mass density of the grain.

Combining Eqs. (44) and (46) we can write the erosion rate of the dust mass as it slows down through the gas as

$\frac{\mathrm{d} m_{\mathrm{gr}}}{\mathrm{d} v_{\mathrm{gr}}}=\frac{m_{\mathrm{gr}}}{v_{\mathrm{gr}}} \frac{m_{\mathrm{sp}}}{m_{\mathrm{H}}} \frac{2}{\gamma} \frac{\sum_{i} n_{i} Y_{i}(E)}{\sum_{i}\left(m_{i} / m_{\mathrm{H}}\right) n_{i}}$,

which can readily be solved for the grain mass $m_{\mathrm{gr}}\left(v_{\mathrm{gr}}\right)$, i.e.

$\frac{m_{\mathrm{gr}}\left(v_{\mathrm{gr}}\right)}{m_{\mathrm{gr}}(0)}=\exp \left[\frac{2}{\gamma} \frac{m_{\mathrm{sp}}}{m_{\mathrm{H}}} \int_{v_{\mathrm{gr}}(0)}^{v_{\mathrm{gr}}(t)} \frac{\sum_{i} n_{i} Y(E)}{\sum_{i}\left(m_{i} / m_{\mathrm{H}}\right) n_{i}} \frac{\mathrm{d} v_{\mathrm{gr}}}{v_{\mathrm{gr}}}\right]$, 
where $m_{\mathrm{gr}}(0)$ and $v_{\mathrm{gr}}(0)$ are the initial grain mass and velocity, respectively, and $m_{\mathrm{gr}}\left(v_{\mathrm{gr}}\right)$ is the grain's mass at velocity $v_{\mathrm{gr}}(t)$.

The column density of gas traversed by a grain is

$N_{\text {gas }}(t)=n_{\text {gas }} \int_{v_{\mathrm{gr}}(0)}^{v_{\mathrm{gr}}(t)} v_{\mathrm{gr}}\left(t^{\prime}\right) \mathrm{d} t^{\prime}$.

Using Eq. (46), we obtain that

$$
\begin{aligned}
\frac{\mathrm{d} N_{\mathrm{gas}}\left(v_{\mathrm{gr}}\right)}{\mathrm{d} v_{\mathrm{gr}}} & =\left[\frac{\mathrm{d} N_{\mathrm{gas}}\left(v_{\mathrm{gr}}\right)}{\mathrm{d} t}\right]\left[\frac{\mathrm{d}\left(v_{\mathrm{gr}}\right)}{\mathrm{d} t}\right]^{-1}, \\
& =\left(n_{\mathrm{gas}} v_{\mathrm{gr}}\right) \frac{4}{3 \gamma} \frac{a_{\mathrm{gr}}(t)}{v_{\mathrm{gr}}^{2}} \frac{\rho_{\mathrm{gr}}}{\sum_{i} \rho_{i}}
\end{aligned}
$$

where $\rho_{i} \equiv n_{i} m_{i}$.

Defining a characteristic slowing down time, $\tau_{\text {sd }}$ as

$\tau_{\mathrm{sd}} \equiv \frac{4}{3}\left[\frac{a_{\mathrm{gr}}(0)}{v_{\mathrm{gr}}(0)}\right]\left(\frac{\rho_{\mathrm{gr}}}{\sum_{i} \rho_{i}}\right)$,

we obtain that

$\frac{\mathrm{d} N_{\mathrm{gas}}\left(v_{\mathrm{gr}}\right)}{\mathrm{d} v_{\mathrm{gr}}}=\frac{1}{\gamma}\left[\frac{a_{\mathrm{gr}}(t)}{a_{\mathrm{gr}}(0)}\right]\left[\frac{v_{\mathrm{gr}}(0)}{v_{\mathrm{gr}}(t)}\right] \tau_{\mathrm{sd}} \sum_{i} n_{i}$,

where $n_{\mathrm{gas}} \equiv \sum_{i} n_{i}$. Since $\left[a_{\mathrm{gr}}(t) / a_{\mathrm{gr}}(0)\right]=\left[m_{\mathrm{gr}}(t) / m_{\mathrm{gr}}(0)\right]^{1 / 3}$ Eq. (52) can be integrated to give

$N_{\mathrm{gas}}\left(v_{\mathrm{gr}}\right)=\frac{1}{\gamma} N_{0} \int_{v_{\mathrm{gr}}(0)}^{v_{\mathrm{gr}}} F\left(v_{\mathrm{gr}}^{\prime}\right) \frac{\mathrm{d} v_{\mathrm{gr}}^{\prime}}{v_{\mathrm{gr}}^{\prime}}$,

where

$F\left(v_{\mathrm{gr}}^{\prime}\right)=\exp \left[\frac{2}{3 \gamma} \frac{m_{\mathrm{sp}}}{m_{\mathrm{H}}} \int_{v_{\mathrm{gr}}(0)}^{v_{\mathrm{gr}}^{\prime}} \frac{\sum_{j} n_{j} Y(E)}{\sum_{i}\left(m_{i} / m_{\mathrm{H}}\right) n_{i}} \frac{\mathrm{d} v}{v}\right]$

and where $N_{0}$ is the column density, defined as

$N_{0} \equiv v_{\mathrm{gr}}(0) \tau_{\mathrm{sd}} \sum_{i} n_{i}=\frac{m_{\mathrm{gr}}(0)}{\pi a_{\mathrm{gr}}^{2}(0)} \frac{\sum_{i} n_{i}}{\sum_{i} \rho_{i}}$,

so that the mass of gas contained within the volume swept up by the grain is equal to the initial mass of the grain if it was not eroded by the gas.

Figure 10 shows which fraction of the initial mass of a dust grain survives the effect of kinetic sputtering. The mass fraction, represented as a function of the initial velocity of the grain $v_{\mathrm{gr}}(0)$, has been calculated after the grain has slowed down to zero, assuming $n_{\text {gas }}=n_{\text {cloud }}=100 \mathrm{~cm}^{-3}$. The mass fraction does not depend on the initial grain size, since the same atoms that slow the grain down are also eroding it by sputtering.

To calculate the time $t_{\mathrm{esc}}$ required by a dust grain to traverse a column density of gas $N_{\text {gas }}$, we use the expression $\mathrm{d} N_{\text {gas }}=$ $n_{\text {gas }} v_{\mathrm{gr}}(N) \mathrm{d} t$, from which we derive

$t_{\mathrm{esc}}=\int_{0}^{N_{\mathrm{gas}}} \frac{\mathrm{d} N^{\prime}}{n_{\mathrm{gas}} v_{\mathrm{gr}}\left(N^{\prime}\right)}$,

where we obtained $v_{\mathrm{gr}}\left(N^{\prime}\right)$ numerically from Eq. (53). Figure 11 is a contour diagram depicting $t_{\mathrm{esc}}$ as a function of the dust grains radius and column density of gas traversed. We calculated the curves for the initial velocity $v_{\mathrm{gr}}(0)=v_{\text {cloud }}=160 \mathrm{~km} \mathrm{~s}^{-1}$. This is appropriate for Cas A, where the cloud shock is highly radiative, therefore the post-shock gas is compressed by a factor $\gg 4$ and
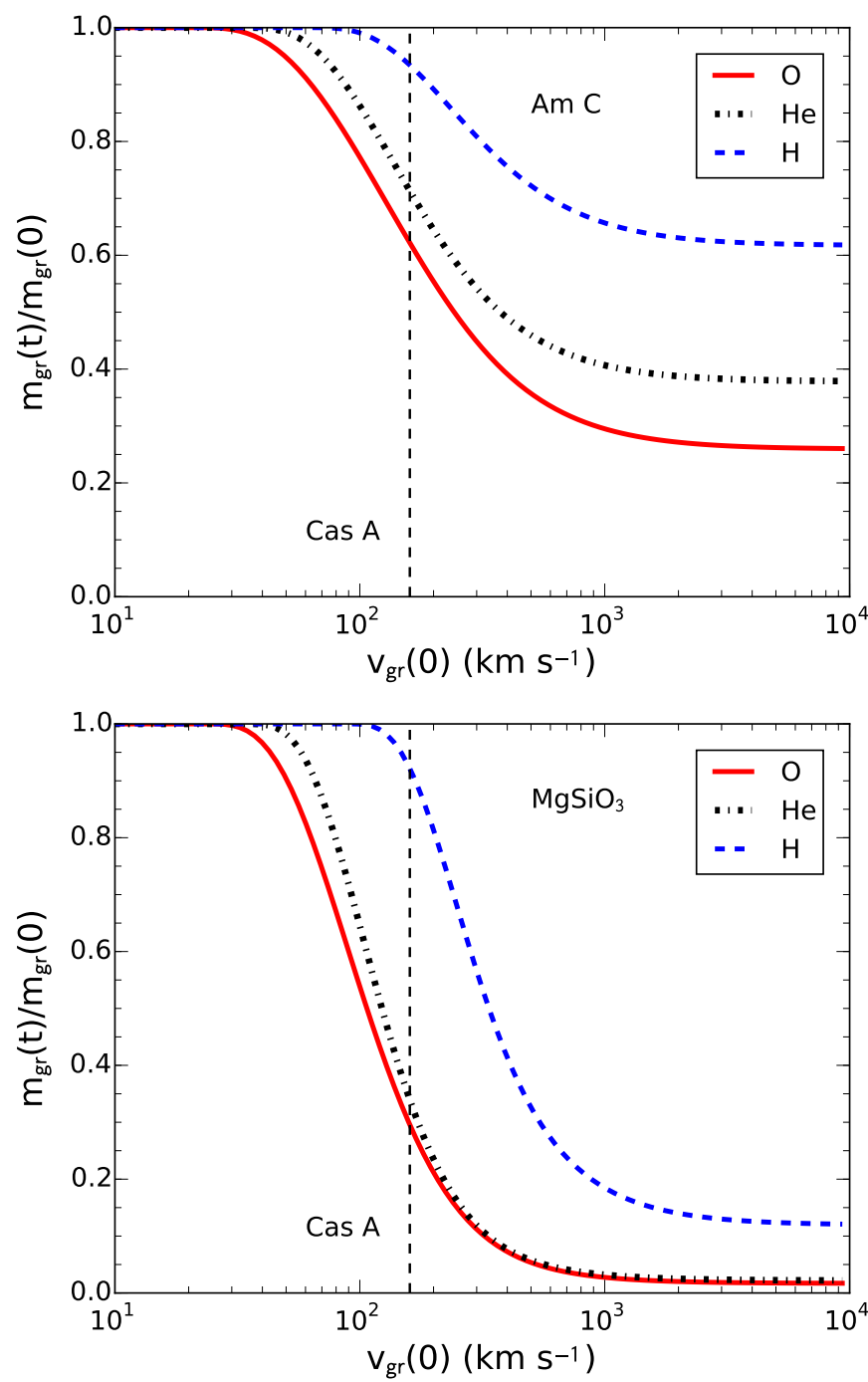

Fig. 10. Mass fraction of a carbon (top panel) and $\mathrm{MgSiO}_{3}$ (bottom panel) dust grain surviving the erosion by kinetic sputtering as a function of its initial velocity $v_{\mathrm{gr}}(0)$, as it traverses a gas of pure $\mathrm{H}, \mathrm{He}$, and $\mathrm{O}$ composition with density $n_{\text {gas }}=n_{\text {cloud }}=100 \mathrm{~cm}^{-3}$. The vertical line indicates the initial grain velocity for $\operatorname{Cas} \mathrm{A}, v_{\mathrm{gr}}(0)=v_{\text {cloud }}=$ $160 \mathrm{~km} \mathrm{~s}^{-1}$.

the relative gas-grain velocity is close to $v_{\text {cloud }}$ (in an adiabatic shock the relative velocity would be $\left.(3 / 4) v_{\text {cloud }}\right)$.

Given an initial dust grain velocity, $v_{\mathrm{gr}}(0)$, the amount of destruction taking place in a clump depends on the column density traversed by the grains after the passage of the shock, which in turns depends on the size of the grains and their location in the cloud. The grains, because of their large inertia, move ballistically through the shock, slipping through the gas at a relative velocity close to $v_{\text {cloud }}$. We assume that the shock is planar, so that all the shocked dust grains move perpendicular to the shock front. Big grains sitting close to the edge of the clumps are able to escape without experiencing substantial sputtering, while smaller grains located deeper inside the cloud are eroded and/or stopped before reaching the surface.

In Appendix B we report an estimate of the amount of kinetic sputtering occurring in the clumps based on a statistical approach. However, this approach is only valid for the largest grain sizes. A Monte Carlo approach is required to properly evaluate the effect of inertial sputtering in the ejecta clouds, which takes 

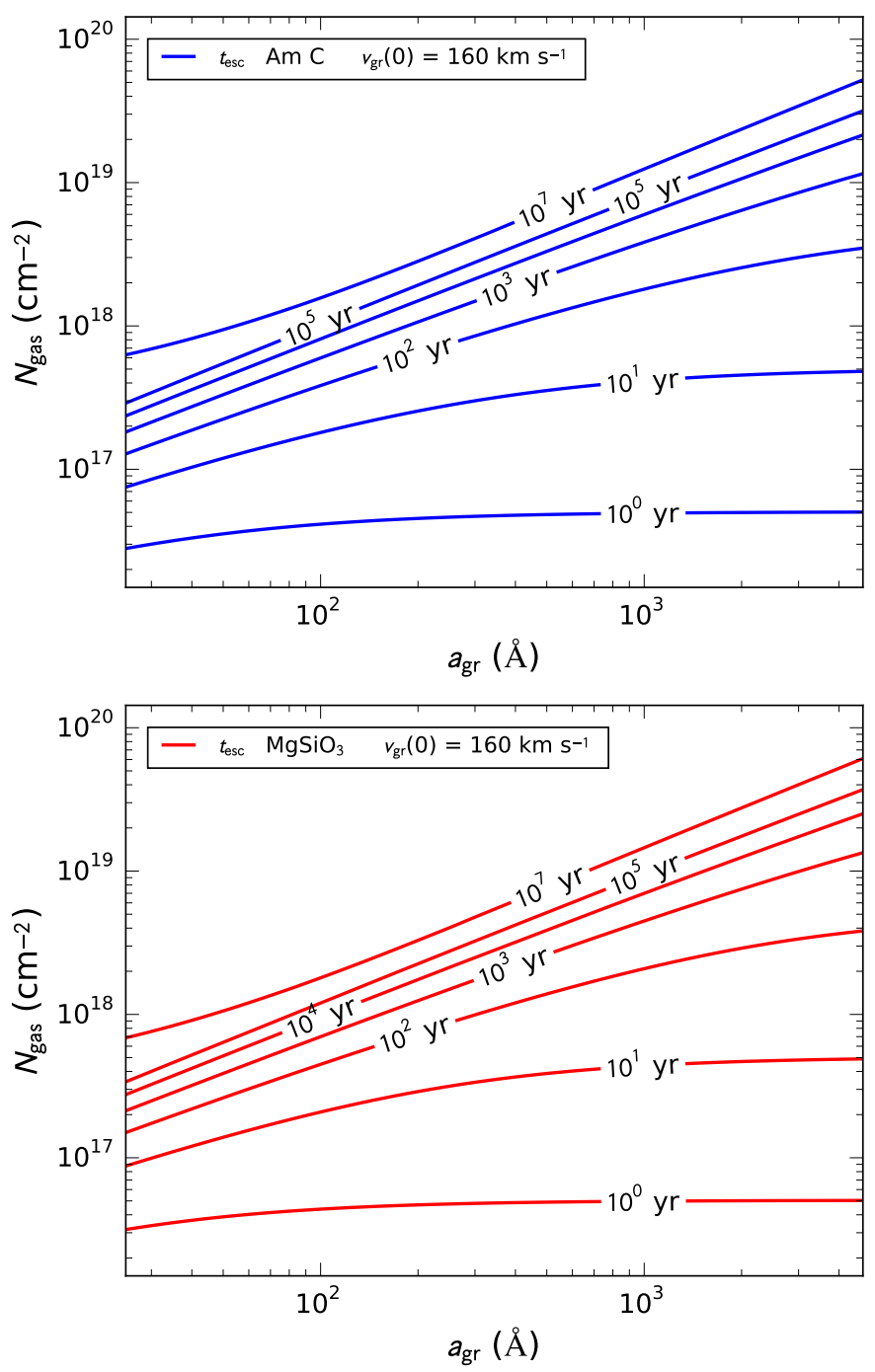

Fig. 11. Contour plot showing the time $t_{\text {esc }}$ required by a dust grain with initial velocity $v_{\mathrm{gr}}(0)=160 \mathrm{~km} \mathrm{~s}^{-1}$ to traverse a column density of gas $N_{\text {gas }}$, as a function of the grain radius $a_{\mathrm{gr}}$.

all grains sizes and positions inside the clumps into account. Methods and results of our simulation are discussed in Sect. 7.

\section{Clump survival: Instabilities and evaporation}

Inertial sputtering occurs in the ejecta clouds and the corresponding amount of destruction depends on how long the grains stay inside the clouds. In the present case, we assume that the residence time depends on three phenomena. The first is the capability of the grains to escape from the cloud owing to their ballistic velocities; we discussed this in Sect. 4. The two other phenomena that we consider are the dynamical fragmentation of the clouds and the thermal evaporation of the clouds, which we discuss below.

The cloud crushing time, $t_{\mathrm{cc}} \equiv R_{\text {cloud }} / v_{\text {cloud }}$ (Klein et al. 1994), provides an indication of the dynamical time required for the cloud shock to fragment the cloud. For $R_{\text {cloud }}=7.5 \times 10^{15} \mathrm{~cm}$ and $v_{\text {cloud }}=160 \mathrm{~km} \mathrm{~s}^{-1}$, we obtain $t_{\mathrm{cc}}=15 \mathrm{yr}$. For the destruction time, $t_{\text {dest }}$, we adopt the value of $3.5 t_{\mathrm{cc}}$ found by Klein et al. (1994) for density contrasts between 10 and 100 (we have 100). This destruction time is defined as the time at which the mass of the core of the cloud has been reduced to a fraction $1 / e$ of the initial cloud mass; it is assumed that the cloud develops a core-plume structure following the passage of the shock. We assume that after $t_{\text {dest }}=3.5 t_{\mathrm{cc}}$ the ejecta cloud is destroyed and dispersed. At that moment, the fresh dust still residing in the cloud will have been released into the smooth ejecta.

A competing process for the disruption of the clumps is thermal evaporation. Thermal evaporation occurs because electron thermal conduction transfers heat from the hot smooth ejecta to the outer layers of the colder clouds. The heated material has an excess of pressure, which then drives an outflow. Cowie $\&$ McKee (1977) examined the steady evaporation of spherical clouds including the effects of saturation of the conductive heat flux. Saturation of the heat flux occurs when the heat flux predicted based on the Spitzer conductivity and the temperature gradient exceeds that which can be carried by the electrons given their density and thermal speed. Dalton \& Balbus (1993) found an analytical solution for cloud evaporation that includes cases of highly saturated conduction which applies for the Cas A knots. Dalton \& Balbus (1993) ignore radiative cooling, which may be important at the high densities and enormous cooling coefficients in the Cas A clouds. However, the contribution of radiative processes decreases progressively with increasing saturation. For the present work, we decided to focus on the effects of saturation and we will include radiative evaporation in a follow-up paper. Using the results from Dalton \& Balbus (1993) for the relation between the saturation parameter, $\sigma_{0}$, and mass loss rate, $\dot{M}$ and taking the highly saturated limit we find

$\dot{M}\left(M_{\odot} / \mathrm{Myr}\right)=1.978 \times 10^{13} \mu^{7 / 12}\left(P / 10^{4} k_{\mathrm{B}}\right)^{5 / 6} R_{\text {cloud }}^{11 / 6}(\mathrm{pc})$,

where $P$ is the pressure in the hot medium, $R_{\text {cloud }}$ is the cloud radius (in pc), and $\mu$ is the mean mass per particle in $\mathrm{g}$. There is no explicit dependence on temperature, although there is a dependence on pressure, which depends on the temperature if density is held constant as it is in Fig. 11. For a cloud of pure $\mathrm{O}^{5+}, \mu=4.45 \times 10^{-24} \mathrm{~g}$. We take as the evaporation timescale the quantity $t_{\text {sat }}=M_{\text {cloud }} / \dot{M}$, which is then written as

$t_{\text {sat }}=4.420 n_{\text {cloud }}\left(P / 10^{4} k_{\mathrm{B}}\right)^{-5 / 6} R_{\text {cloud }}^{7 / 6}(\mathrm{pc}) \mathrm{Myr}$,

where $n_{\text {cloud }}$ is the cloud space density (in $\mathrm{cm}^{-3}$ ). The high pressure in the remnant reduces the evaporation timescale well below typical ISM values, but it is still generally larger than the cloud crushing time in this context. Thus the cloud is torn apart before it can evaporate, although evaporation could play a role in destroying some cloud fragments.

The timescales discussed above are shown in Fig. 12 as a function of the diameter of the clouds, for carbon and silicate grains. We calculated the evaporation time $t_{\text {sat }}$ for the two temperatures $T=10^{7} \mathrm{~K}$ and $T=10^{8} \mathrm{~K}$. For the escape time $t_{\mathrm{esc}}$, the two curves correspond to two grain sizes: $2500 \AA$ (the upper limit of our grain size distribution, see Sect. 7) and $1000 \AA$, an intermediate value. For each cloud diameter, the value of $t_{\mathrm{esc}}$ in the figure represents the time required to cross that diameter, which is the maximum path that a grain has to traverse to escape the cloud. Therefore, $t_{\mathrm{esc}}$ is the maximum escape time. We calculated both $t_{\text {esc }}$ and $t_{\text {dest }}$ assuming $v_{\mathrm{gr}}(0)=v_{\text {cloud }}=160 \mathrm{~km} \mathrm{~s}^{-1}$, where $v_{\mathrm{gr}}(0)$ is the initial velocity of the grains. The value $160 \mathrm{~km} \mathrm{~s}^{-1}$ is the cloud shock velocity corresponding to the constant reverse shock velocity in the ejecta core, which is also the lowest velocity reached by the reverse shock in Cas A (see Fig. 4). From the definition of $t_{\text {dest }}=3.5 t_{\mathrm{cc}}=R_{\text {cloud }} / v_{\text {cloud }}$ and $t_{\text {esc }}$ (Eq. (56)) it follows, therefore, that for each cloud size, the values of $t_{\text {dest }}$ and $t_{\text {esc }}$ shown in Fig. 12 are upper limits. 

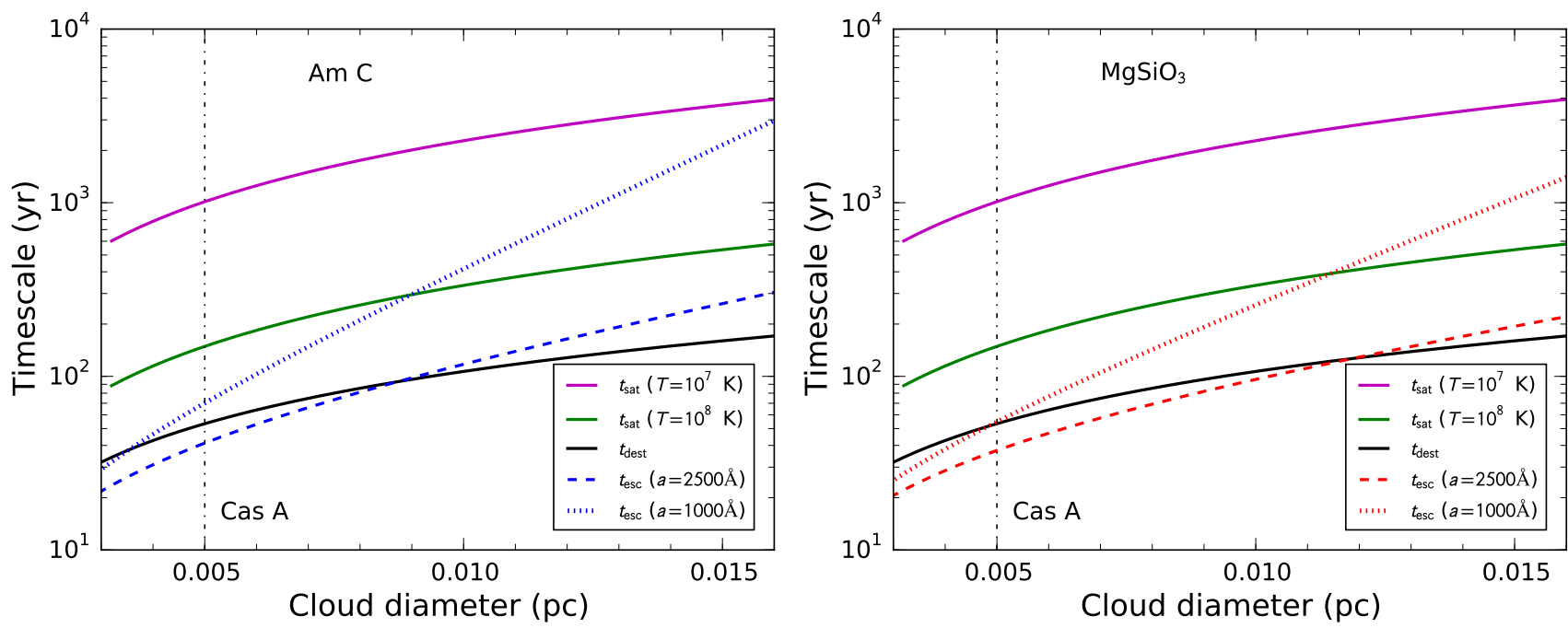

Fig. 12. Comparison between timescales relevant for the processing of carbon (left panel) and silicate (right panel) dust grains in an ejecta cloud: cloud destruction time $t_{\text {dest }}$, cloud evaporation time $t_{\text {sat }}$, calculated for two temperatures of the gas surrounding the clouds, and dust escape time $t_{\text {esc }}$, calculated for two grain sizes. Both $t_{\mathrm{esc}}$ and $t_{\text {dest }}$ has been calculated assuming $v_{\mathrm{gr}}(0)=v_{\text {cloud }}=160 \mathrm{~km} \mathrm{~s}^{-1}$. All timescales are represented as a function of the diameter of the clump. The vertical line indicates the diameter adopted for the ejecta clouds in Cas A. See text for details.

From Fig. 12 and from the considerations above it follows that, for Cas A, the trade is between the escape time and the destruction time. For both carbon and silicate dust, grains that are sufficiently big are able to escape before the cloud is disrupted, even if they are located far from the surface. These fugitive grains experience thermal sputtering in the hot phase of the ejecta before their counterparts ejected at time $t_{\text {dest }}$. The injection of dust in the hot medium is a continuous process depending on the grain size and position inside the clouds. To properly investigate such a process, an analytical approach is not applicable, therefore we decided to perform a Monte Carlo simulation; this is described in Sect. 7.

\section{Grain destruction by the reverse shock: Thermal sputtering}

After a dust grain is ejected from a cloud, because of its ballistic velocity or because of the destruction of the cloud, it will find itself in the smooth hot ejecta. Here the temperature is much higher than in the clouds, therefore the dominant erosion mechanism is thermal sputtering, where the velocity of the ions is determined by the temperature of the shocked gas (thermal motion). The effect of thermal sputtering has been calculated for two temperatures of the smooth ejecta: $T \sim 10^{8} \mathrm{~K}$ (from Eq. (35)) and $T \sim 10^{7} \mathrm{~K}$ (for comparison).

The variation of the grain radius, $a_{\mathrm{gr}}$, due to thermal sputtering is given by Eq. (4.20) in Tielens et al. (1994),

$\frac{1}{n_{\mathrm{H}}} \frac{\mathrm{d} a_{\mathrm{gr}}}{\mathrm{d} t}=\frac{m_{\mathrm{sp}}}{\rho_{\mathrm{gr}}} \sum A_{i}\left\langle Y_{i} v\right\rangle$

where $\left\langle Y_{i} v\right\rangle$ is the sputtering yield of ion $i$ averaged over the Maxwellian distribution ( $Y_{i}$ as in Sect. 4), $v$ is the velocity of the ions, $n_{\mathrm{H}}$ is the hydrogen number density of the smooth ejecta, and $A_{i}$ is the abundance of ion $i$ with respect to hydrogen. As discussed in Sect. 3.2, we assume for the smooth ejecta a mixture of $\mathrm{O}, \mathrm{Ne}, \mathrm{Mg}, \mathrm{Si}, \mathrm{S}, \mathrm{Ar}$, and $\mathrm{Fe}$ as determined by Hwang \& Laming (2012). Because of the compression due to the shock, the density of the smooth ejecta must be increased by a factor of four with respect of its pre-shock value. The Monte Carlo approach used to evaluate thermal sputtering across the remnant is described in Sect. 7.

\section{A Monte Carlo approach to evaluate kinetic and thermal sputtering: Methods and results}

For each ejecta cloud, we consider a population of $3 \times 10^{6}$ particles of each dust grain type (carbon and silicate) with a given size distribution and homogeneously distributed over the entire volume of the cloud. We follow the journey of each particle to determine its final size (and consequently mass) as a result of kinetic sputtering (in the ejecta clumps) and thermal sputtering (in the smooth ejecta). As described in Sect. 3, we adopt the classical MRN expression for the dust grain size distribution (Mathis et al. 1977).

Following the encounter with the reverse shock, the dust grains inside the cloud acquire a relative velocity with respect to gas. This velocity decreases asymptotically to zero because of gas-grain collisions, which at the same time are responsible for the progressive erosion of the grains (kinetic sputtering). Depending on their initial size and position inside the cloud, some of the grains are able to escape into the smooth ejecta before the cloud gets disrupted. These fugitive grains therefore experience thermal sputtering for a longer time than their counterparts trapped inside the cloud.

The basic equation governing our Monte Carlo simulation, which allows us to calculate the final radius $a_{\text {final }}$ of a dust grain that experienced the processing previously described, is the following:

$a_{\text {final }}(t)=K_{\mathrm{s}}(t) a_{\text {initial }}-T_{\mathrm{s}} \times\left[\frac{1}{\left(t_{\mathrm{r}}+t_{\text {inj }}\right)^{2}}-\frac{1}{t^{2}}\right]$.

The simulation starts when the reverse shock touches the outer layer of ejecta ( $\alpha=1, \sim 0.9$ years after explosion, see Fig. 1), and stops when the reverse shock reaches the centre of the remnant ( $\alpha=0, \sim 8000$ years after explosion). In Eq. (60), $a_{\text {initial }}$ is the size of the grain before processing; $t_{\mathrm{r}}$ is the time when the reverse shock hits an ejecta cloud located in a layer $\alpha$ (see Sect. 2.2); and $t_{\text {inj }}$ is the time interval (starting from $t_{\mathrm{r}}$ ) after which the grains 


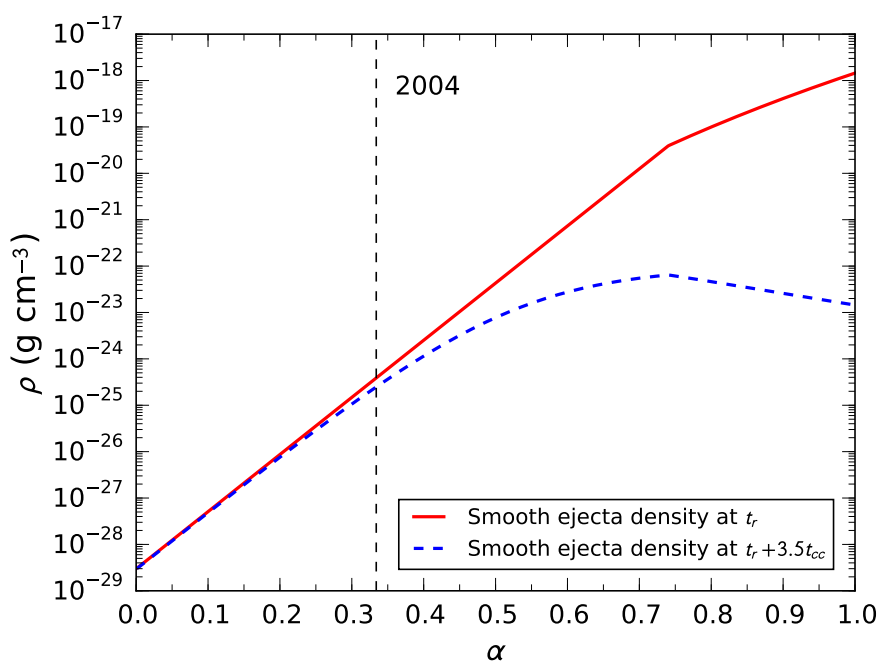

Fig. 13. Density structure of the smooth ejecta as a function of the parameter $\alpha$. Solid red line: density at the time $t_{\mathrm{r}}$ when the layer $\alpha$ encounters the reverse shock (same as Fig. 2); dashed blue line: density at the time $t_{\mathrm{r}}+3.5 t_{\mathrm{cc}}$, when the ejecta cloud is dispersed and the dust still in there is injected into the smooth phase. Vertical line as in Fig. 1.

are expelled from the clouds and injected into the smooth ejecta and is given by the relation $t_{\mathrm{inj}}=\operatorname{Min}\left(t_{\mathrm{esc}}, t_{\mathrm{dest}}\right)$. For each layer, we computed $\alpha, t_{\text {esc }}$ and $t_{\text {dest }}$ using the value for $v_{\mathrm{gr}}(0)=v_{\text {cloud }}$ corresponding to that layer and derived from $v_{\mathrm{r}}$ (see Fig. 4), and we appropriately calculated $t_{\mathrm{esc}}$ from Eq. (56) for each grain size and position inside the cloud (Sect. 4).

The term $K_{\mathrm{s}}(t)$ is the reduction factor of the grain radius due to kinetic sputtering. We derived this term from Eq. (48) (to the power $1 / 3$ to convert from mass to radius), where we derived the final velocity of the grain at time $t, v_{\mathrm{gr}}(t)$, inverting Eq. (53), and the column density $N_{\mathrm{gr}}$ comes from inversion of Eq. (56). It is useful to remember here that the effect of kinetic sputtering is of reducing the mass of each dust grain by the same factor, regardless of its initial size (Eq. (48)). Because of the relationship between grain mass and grain radius, the same statement holds true for the grain radius as well, therefore $a_{\text {final }}(t) / a_{\text {initial }}$ is independent from $a_{\text {initial }}$. Kinetic sputtering is switched OFF for $t>t_{\text {inj }}$, when the grains leave the clouds.

The term $T_{\mathrm{s}} \times[\ldots]$ accounts for the effect of thermal sputtering; this process removes a layer of equal thickness from each grain, regardless of its size. This implies of course that the smaller grains are more affected than the bigger grains. We obtain

$T_{\mathrm{s}}=\frac{n(t) t^{3}}{2} \times\left(\frac{m_{\mathrm{sp}}}{\rho_{\mathrm{gr}}} \sum A_{i}\left\langle Y_{i} v\right\rangle\right)$,

where the term in parenthesis comes from Eq. (59) and the density of the expanding smooth ejecta, $n(t)$ has been calculated from Eqs. (13) and (19). Because of the expansion of the supernova remnant, the density of the smooth ejecta changes while inertial sputtering is occurring inside the clouds. Therefore, the dust is progressively injected into a medium whose density changes continuously. Figure 13 illustrates this phenomenon. The solid red line represents the density of the smooth ejecta at the time $t_{\mathrm{r}}$ when each layer $\alpha$ encounters the reverse shock (same curve as in Fig. 2). The dashed blue line shows the density of the same layer $\alpha$ but at the time $t_{\mathrm{r}}+3.5 t_{\mathrm{cc}}$, when the clumps in that layer are destroyed and the dust still residing in them is released into the smooth phase of the ejecta. In the outer layers ( $\alpha$ close to one) the difference is very pronounced, and decreases progressively towards the inner layers ( $\alpha$ close to zero). This has to be taken into account to properly evaluate the effect of thermal sputtering, which strongly depends on the density of the medium.

Using Eq. (60) we have evaluated the fraction of dust which is expected to survive processing while the reverse shock progresses towards the centre of the remnant, expressed in terms of the parameter $\alpha$. We assume a homogeneous distribution of ejecta clouds in each layer. The fraction of surviving dust is calculated for the entire remnant, i.e. this calculation includes not only the amount of dust remaining after processing down to (but excluding) layer $\alpha$, but also the unprocessed dust located inside and inwards of layer $\alpha$. Because we stopped our simulation at $\alpha=0$, we did not consider further processing of the dust due to the secondary blast-wave shock which generates when the reverse shock bounces at the centre of the remnant (Truelove \& McKee 1999).

Our results are shown in Fig. 14, depicting the evolution of the fraction of surviving dust, $M_{\text {residual }} / M_{\text {init }}$ as a function of $\alpha$ for $\mathrm{Am} \mathrm{C}$ and $\mathrm{MgSiO}_{3}$ grains. In each panel, the top curve illustrates the effect of kinetic sputtering only; this sputtering occurs inside the clumps. The two other curves refer to the combined effect of kinetic and thermal sputtering, and this latter is evaluated for two temperatures of the smooth ejecta: $10^{7} \mathrm{~K}$ and $10^{8} \mathrm{~K}$. We notice that the effect of sputtering is important over the entire remnant. At the end of the processing $(\alpha=0)$, kinetic sputtering has contributed significantly to particle erosion, removing $20 \%$ of the mass of carbon grains and $40 \%$ of the mass of silicate grains (note that the plots are in semi-logarithmic scale). Thermal sputtering reduces the final amount of surviving dust even more dramatically.

Silicate grains appear more fragile than carbonaceous grains. This behaviour results from the combination of different factors: their slightly higher sputtering yield; the average mass of the sputtered particles, almost twice that for Am C (23 amu versus $12 \mathrm{amu}$ ); and the tendency of a larger fraction of silicate grains to escape the ejecta cloud before disruption, which exposes them to thermal sputtering for a longer time.

To provide a feeling for the preferred grain sizes of the surviving dust, in Fig. 15 we plot the dust mass distribution as a function of the grain radius, which is normalized to the total surviving mass for different values of $\alpha$, i.e.

$f(a)=\frac{1}{M(\alpha)} \frac{\mathrm{d} m}{\operatorname{dlog} a}(a) \quad$ with $M(\alpha)=\int \mathrm{d} \log a \frac{\mathrm{d} m}{\operatorname{dlog} a}$,

where $M(\alpha) \equiv M_{\text {residual }}$ (Fig. 14). The initial mass distribution of carbon and silicate dust ( $\alpha=1$, no processed layers) is modified into the final mass distribution ( $\alpha=0$, all layers processed). We evaluated the mass distribution for two temperatures of the smooth ejecta, $T=10^{7} \mathrm{~K}$ and $T=10^{8} \mathrm{~K}$, and the resulting curves are almost indistinguishable. We decided to show the evolution corresponding to $T=10^{8} \mathrm{~K}$, but the curves are indeed representative of the temperature range $10^{7}-10^{8} \mathrm{~K}$. Both dust types show a progressive modification towards their final distributions, which are very similar and cover the original size range (50-2500 $)$ ) with a small leakage down to $30 \AA$. The differences reflect the tendency of $\mathrm{MgSiO}_{3}$ grains to dissociate more easily than carbonaceous grains.

\section{Discussion and conclusions}

In Table 4 we report the percentage of surviving dust mass for $\mathrm{Am} \mathrm{C}$ and $\mathrm{MgSiO}_{3}$ grains evaluated at two specific times during 

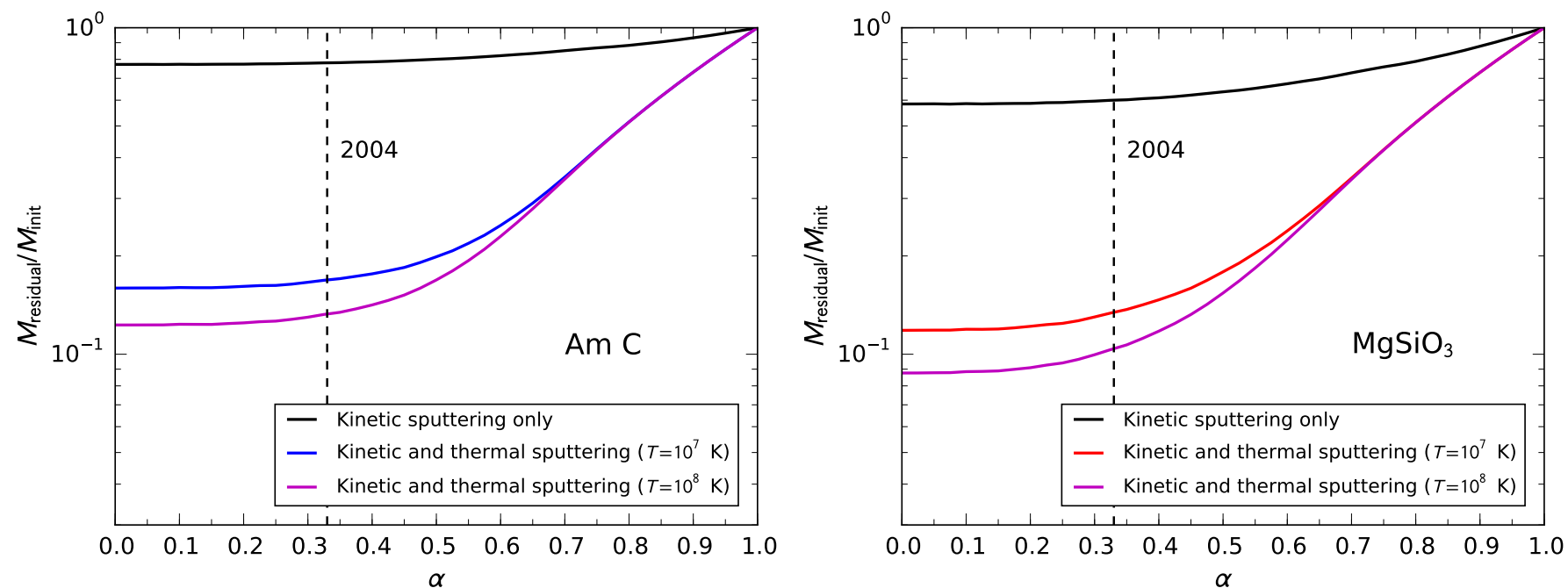

Fig. 14. Fraction of surviving carbon (left panel) and silicate dust (right panel) over the entire Cas A remnant as a function of $\alpha$. For each value of $\alpha$ the curve provides the fraction of remaining dust, which is given by the grains able to survive processing located in the outer layers down to $\alpha$ plus the unprocessed dust residing in the inner layers. The three curves show the effect of kinetic sputtering only and of kinetic +thermal, the latter evaluated for two temperatures of the smooth ejecta: $T=10^{7} \mathrm{~K}$ and $T=10^{8} \mathrm{~K}$.
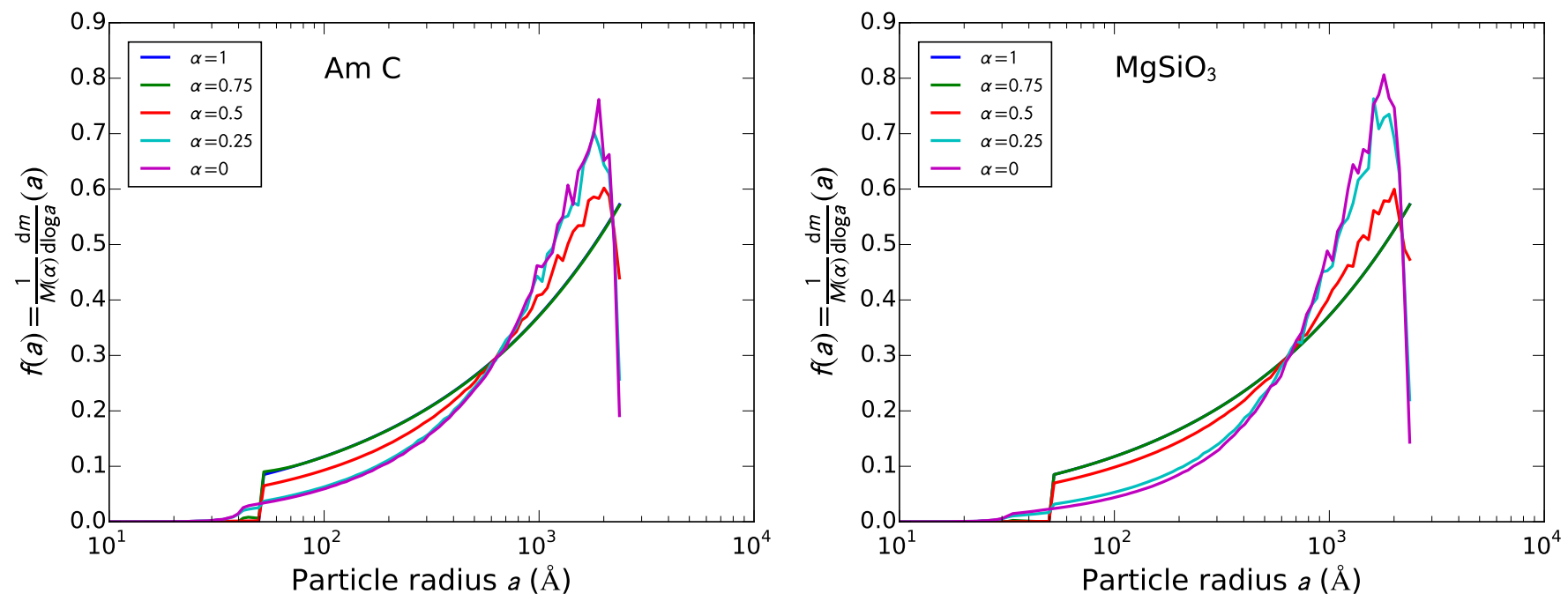

Fig. 15. Surviving mass distribution as a function of the grain radius from Eq. (62), for carbon (left panel) and silicate dust (right panel), evaluated for temperatures of the smooth ejecta between $10^{7} \mathrm{~K}$ and $10^{8} \mathrm{~K}$. The distributions were normalized to the total surviving dust mass (with $M(\alpha) \equiv$ $M_{\text {residual }}$ ) so that the area under each curve equals 1 .

the evolution of the remnant: in 2004 ( $\alpha=0.33$ ), when some of the astronomical observations used in this work were carried out, and after the reverse shock reached the centre of the remnant $(\alpha=0)$.

In 2004, the amounts of surviving dust are of the same order of magnitude, but we can notice the difference between the two type of grains and the increased destructive effect of the higher temperature (see Fig. 14). Arendt et al. (2014) estimated the amount of dust that should be currently present in Cas A: $\lesssim 0.1 M_{\odot}$ (cold unshocked ejecta) $+\sim 0.04 M_{\odot}$ (shocked circumstellar/interstellar and ejecta regions). If we assume that this amount of dust represents the percentage of surviving mass that we calculate, we can derive the corresponding amount of dust that Cas A should have produced. Taking a representative value of $13.5 \%$, to average over dust type and gas temperature, we obtain an initial mass of dust of about $1 M_{\odot}$, which reduces to $\sim 0.8 M_{\odot}$ if only silicates are considered. These values are larger than the current estimate of $0.1-0.5 M_{\odot}$ (Todini \& Ferrara 2001; Schneider et al. 2004; Cherchneff \& Dwek 2010; Nozawa et al. 2010). The situation is similar after the reverse shock has swept the entire cavity of the SNR, reaching the centre $(\alpha=0)$. This is not surprising because, after 2004, the fraction of surviving dust mass tends to reach a plateau, as shown in Fig. 14.

We compared our findings with the results from Bianchi \& Schneider (2007), calculated for homogeneous (smooth) ejecta with a post-shock temperature of the order of $10^{7}-10^{8} \mathrm{~K}$ expanding in a homogeneous medium, for a progenitor star of 15$25 M_{\odot}$ and a density of the surrounding ISM of $10^{-24} \mathrm{~g} \mathrm{~cm}^{-3}$. These values are the closest to the parameters that we adopt for Cas A: same mass range for the progenitor star and a circumstellar medium with density $n_{0}=2.071 \mathrm{H}$ atom $\mathrm{cm}^{-3}$, corresponding to $\rho_{0}=3.6 \times 10^{-24} \mathrm{~g} \mathrm{~cm}^{-3}$. We evaluated the effect of the two 
Table 4. Percentage of dust mass, $M_{\text {residual }} / M_{\text {init }}$, surviving the passage of the reverse shock in Cas A, evaluated in two different stages of supernova evolution identified by the corresponding values of $\alpha$.

\begin{tabular}{c|c|c|c|c|c}
\hline \hline$T^{a}$ & \multicolumn{2}{|c|}{$\alpha=0.33^{b}$} & \multicolumn{2}{c|}{$\alpha=0^{c}$} & $\mathrm{BSO7}^{d}$ \\
$(K)$ & $\mathrm{Am} \mathrm{C}$ & $\mathrm{MgSiO}_{3}$ & $\mathrm{Am} \mathrm{C}$ & $\mathrm{MgSiO}_{3}$ & $\mathrm{C}+\mathrm{Fe}_{3} \mathrm{O}_{4}$ \\
\hline$\sim 10^{7}$ & $16.9 \%$ & $13.4 \%$ & $15.9 \%$ & $11.8 \%$ & \\
$\sim 10^{8}$ & $13.3 \%$ & $10.4 \%$ & $12.3 \%$ & $8.7 \%$ & $\sim 7.5 \%$ \\
\hline
\end{tabular}

Notes. The result from Bianchi \& Schneider (2007) is reported for comparison. ${ }^{(a)}$ Temperature of the smooth ejecta. ${ }^{(b)}$ Corresponding to 2004 (333 yr after explosion). ${ }^{(c)}$ After the reverse shock has reached the centre of the remnant ( $\sim 8000 \mathrm{yr}$ after explosion). ${ }^{(d)}$ Derived from Bianchi \& Schneider (2007): gas temperature between $10^{7}$ and $10^{8} \mathrm{~K}$; surviving mass calculated after the reverse shock has hit the centre of the remnant.

temperature values separately. We assume that the amount of surviving dust calculated by Bianchi \& Schneider (2007), shown in their Fig. 5, refers to a mixture of amorphous carbon and $\mathrm{Fe}_{3} \mathrm{O}_{4}$, whose pre- and post-shock size distributions are reported in their Fig. 4.

From Fig. 5 in Bianchi \& Schneider (2007) we derive that the amount of dust surviving the passage of the reverse shock is around $7.5 \%$. This is around a factor of (1.2-2.1) lower than our findings. Some considerations have to be made when comparing these results. Bianchi \& Schneider (2007) adopted for Cas A homogenous smooth ejecta only (no clumps), expanding into a uniform medium. This choice has two implications. The first is a different velocity profile for the reverse shock than the more realistic case of inhomogeneous smooth ejecta (uniform core + power-law envelope) expanding into a power-law ambient medium, which we considered in this work. The second is that the absence of oxygen-rich, fast-cooling ejecta clumps means that the dust spends all its life in the highly destructive hot smooth ejecta. In our case the grains reside for some time in the protective environment represented by the cold clumps, where they are processed by kinetic sputtering, which is less efficient in destroying the dust.

A comparison of our results with previous work is complicated by a number of factors: the different approaches (numerical or analytical) and descriptions of the supernova ejecta and ambient environment adopted in the various studies, and the different choices for the grain destruction processes, grain composition and size distribution. Following the quantitative description of Dwek (2005), Nozawa et al. (2007) calculated the dust survival in the SN ejecta, exploring various explosion energies, ambient media densities, and grain sizes and composition. Using numerical models, they find that the fraction of the dust mass destroyed ranges from 0.2 to 1.0 , depending on model parameters. Using the analytical model of Truelove \& McKee (1999) and the SN dust composition and size distribution of Todini \& Ferrara (2001), Bianchi \& Schneider (2007) found that only between 2 and $20 \%$ of the dust mass survives the passage of the reverse shock depending on the parameters adopted. Exploring a wide range of dust parameters and ambient gas density profiles, Nath et al. (2008) found that the fractional mass of grains destroyed is $\lesssim 20 \%$, and they provide a detailed discussion of why their results are significantly lower than those found by Nozawa et al. (2007) or Bianchi \& Schneider (2007). Recently, Biscaro \& Cherchneff (2016) derived a dust mass survival fraction between 6 and $11 \%$, using the analytical model of Truelove \& McKee (1999) and the SN dust composition and size distribution of Biscaro \& Cherchneff (2014).
All models discussed above (except Biscaro \& Cherchneff 2016) assumed that the dust grains are embedded in homogeneous ejecta. Silvia et al. $(2010,2012)$ performed 3D numerical simulations of the interaction of planar shocks with dense spherical clumps for a range of shock velocities, clump-ejecta density contrasts, and clump metallicities. Taking the effects of cloud crushing into account, they calculated the amount of grain destruction as a function of their parameter grid using a SN dust compositions with the size distribution calculated by Nozawa et al. (2003). Silvia et al. find a dust mass survival fraction between 7 and $99 \%$ depending on the parameters of the simulation and the dust composition. These results however are not included in an evolutionary model of the reverse shock. Our surviving fractions are significantly below those of Silvia et al. (2010), although the differences in approaches prevent direct comparison.

Our estimate of the surviving dust mass fraction has to be considered a lower limit because we focused on the phenomena able to destroy the dust. We did not consider conditions which could help the dust to survive, for instance we set to zero the differential velocities that grains of different size retain once they have leaved the ejecta clouds. These velocities could allow the dust to escape the shell of shocked ejecta before suffering excessive thermal sputtering. Our present work does not include betatron acceleration of the charged grains induced by the magnetic field nor radiative evaporation of the ejecta clumps. The impact of all these phenomena on the survival of the dust will be evaluated in a follow-up paper.

An interesting fact to point out is that if most of the $\mathrm{C}$ and $\mathrm{Si}$ in the FMKs is in dust, and if the dust destruction in such clumps is mainly due to kinetic sputtering at low temperatures induced by the cloud shock, then there would be little emission in lines such as the UV lines of C III], C IV, Si III] and Si IV or the [Fe II] lines even if the abundances of those elements are substantial. The UV is, of course, not observable in Cas A because of the heavy galactic reddening, but spectra of the SNRs N132D (in the LMC) and 1E0102.2-7219 (in the SMC) are available (Blair et al. 2000). These spectra show some carbon, a little silicon, and some magnesium, providing indicative lower limits to the carbon grain destruction or gas phase abundance.

\section{Summary}

In this work, we approach the still-open question of the survival of freshly formed dust against processing by the reverse shock inside the cavity of supernova remnants, where the dust is actually formed. We have developed analytical or semi-analytical expressions for the relevant quantities, which we implemented into a Monte Carlo simulation. We focus on the specific case of the well-studied supernova remnant Cas A. Our main findings are summarized below.

- Following the seminal work of Truelove \& McKee (1999) further developed by Laming \& Hwang (2003), we derived analytical expressions to describe the evolution of the forward and reverse shock in Cas A, considering inhomogeneous ejecta (uniform core+power-law envelop) expanding into a non-uniform (power-law) ambient medium. This configuration is a closer match to the physical situation of the remnant.

- We adopted a set of parameters that allows us to reproduce the dynamics, and the density and temperature profile of the Cas A ejecta, as deduced by recent observations. In 
particular, we assume that the dust forms in dense clumps immersed in smooth and tenuous ejecta.

- The ejecta in the clumps are heated up by the cloud shock generated by the impact between the clumps and the reverse shock. Because of their high oxygen content, the ejecta cool down quickly to temperatures below $1000 \mathrm{~K}$. Under these conditions, kinetic sputtering is at work.

- For the smooth ejecta, we consider two temperatures: $\sim 10^{8} \mathrm{~K}$ (from our calculations) and $\sim 10^{7} \mathrm{~K}$ (average value from observations). Once the dust grains are injected into the smooth hot ejecta (because of their ballistic velocities or due to cloud destruction), they are processed by thermal sputtering. For the destruction of the clouds, we compared the effects of dynamical instabilities and thermal evaporation.

- We adopted a Monte Carlo approach to derive the mass and size distribution of the processed dust during the progression of the reverse shock inside the remnant. The sputtering of dust is calculated across the remnant and during its evolution, making use of the appropriate profiles that we have derived for relevant quantities, such as the velocity of the reverse shock and the ejecta density.

- Our simulation starts when the reverse shock touches the outer layer of ejecta ( $\alpha=1, \sim 0.9 \mathrm{yr}$ after explosion), and stops when the reverse shock reaches the centre of the remnant ( $\alpha=0, \sim 8000$ yr after explosion).

- The fraction of surviving dust mass decreases while the reverse shocks proceeds towards the centre of the remnant and more ejecta are processed. Silicate grains $\left(\mathrm{MgSiO}_{3}\right)$ are more prone to erosion than amorphous carbon (Am C) grains.

- The effect of rising the temperature of the smooth ejecta from $10^{7} \mathrm{~K}$ to $10^{8} \mathrm{~K}$ becomes noticeable in the inner layers of the ejecta (decreasing values of $\alpha$ ): $\alpha \lesssim 0.55$ for both carbon and silicate dust.

- For 2004 ( $\alpha=0.33)$, we calculate a survival fraction of $16.9 \%$ and $13.3 \%$ for Am C, and of $13.4 \%$ and $10.4 \%$ for $\mathrm{MgSiO}_{3}$, when the grains are immersed in a gas with a temperature of $10^{7} \mathrm{~K}$ and $10^{8} \mathrm{~K}$ respectively. About $0.1 M_{\odot}$ of dust is currently observed in Cas A. If all this dust represents the surviving fraction, then about $0.8-1 M_{\odot}$ of dust must have formed in the ejecta. This suggests that almost all refractory elements condensed onto dust, implying a condensation efficiency that is somewhat higher than in models that predict the formation of $0.1-0.5 M_{\odot}$ of dust in CCSNe ejecta.

- After the reverse shock has reached the centre of the remnant $(\alpha=0)$, we obtain the following survival fraction: $15.9 \%$ for $\mathrm{Am} \mathrm{C}$ and $11.8 \%$ for $\mathrm{MgSiO}_{3}$ for a gas temperature of $10^{7} \mathrm{~K}, 12.3 \%$ for $\mathrm{Am} \mathrm{C}$ and $8.7 \%$ for $\mathrm{MgSiO}_{3}$ when the gas temperature is $10^{8} \mathrm{~K}$. Kinetic sputtering destroys by itself $20 \%$ of the carbon and $40 \%$ of the silicate grains.

The surviving mass fractions depend on the morphology of the ejecta and medium the remnant is expanding into, as well as the composition and size distribution of the grains that formed in the ejecta. Results, therefore, differ for different types of supernova. The framework that we have developed to study dust processing and survival in Cas A can be adapted to other supernova remnants, both young (SN 1985A, the Crab Nebula, Kepler and Tycho) and more evolved (Cygnus Loop).

Acknowledgements. We are grateful to our anonymous referee for the careful reading and valuable suggestions and we would like to thank Martin Laming, Richard Arendt, Mordecai-Mark Mac Low, and Alex Hill for useful and stimulating discussions. E.R.M. wishes to acknowledge the support from a Marie Curie Intra-European Fellowship within the 7th European Community Framework
Programme under project number PIEF-GA-2012-328902 NANOCOSMOS E.D. acknowledges the support of NASA's 13-ADAP13-0094.

\section{References}

Allen, M. G., Groves, B. A., Dopita, M. A., Sutherland, R. S., \& Kewley, L. J. 2008, ApJS, 178, 20

Arendt, R. G., Dwek, E., Kober, G., Rho, J., \& Hwang, U. 2014, ApJ, 786, 55 Asplund, M., Grevesse, N., Sauval, A. J., \& Scott, P. 2009, ARA\&A, 47, 481 Baines, M. J., Williams, I. P., \& Asebiomo, A. S. 1965, MNRAS, 130, 63 Barlow, M. J., Krause, O., Swinyard, B. M., et al. 2010, A\&A, 518, L138 Bertoldi, F., Carilli, C. L., Cox, P., et al. 2003, A\&A, 406, L55

Bianchi, S., \& Schneider, R. 2007, MNRAS, 378, 973

Biscaro, C., \& Cherchneff, I. 2014, A\&A, 564, A25

Biscaro, C., \& Cherchneff, I. 2016, A\&A, 589, A132

Blair, W. P., Morse, J. A., Raymond, J. C., et al. 2000, ApJ, 537, 667

Bocchio, M., Jones, A. P., \& Slavin, J. D. 2014, A\&A, 570, A32

Borkowski, K. J., \& Shull, J. M. 1990, ApJ, 348, 169

Bouchet, P., De Buizer, J. M., Suntzeff, N. B., et al. 2004, ApJ, 611, 394

Cherchneff, I., \& Dwek, E. 2010, ApJ, 713, 1

Chevalier, R. A., \& Fransson, C. 1994, ApJ, 420, 268

Chevalier, R. A., \& Kirshner, R. P. 1978, ApJ, 219, 931

Chevalier, R. A., \& Kirshner, R. P. 1979, ApJ, 233, 154

Chevalier, R. A., \& Oishi, J. 2003, ApJ, 593, L23

Clayton, D. D., \& Nittler, L. R. 2004, ARA\&A, 42, 39

Cowie, L. L., \& McKee, C. F. 1977, ApJ, 211, 135

Dalton, W. W., \& Balbus, S. A. 1993, ApJ, 404, 625

DeLaney, T., \& Rudnick, L. 2003, ApJ, 589, 818

Docenko, D., \& Sunyaev, R. A. 2010, A\&A, 509, A59

Draine, B. T. 2011, Physics of the Interstellar and Intergalactic Medium (Princeton University Press)

Dunne, L., Maddox, S. J., Ivison, R. J., et al. 2009, MNRAS, 394, 1307

Dwek, E. 2005, in The Spectral Energy Distributions of Gas-Rich Galaxies: Confronting Models with Data, eds. C. C. Popescu, \& R. J. Tuffs, AIP Conf. Ser., 761, 103

Dwek, E., \& Arendt, R. G. 1992, ARA\&A, 30, 11

Dwek, E., \& Cherchneff, I. 2011, ApJ, 727, 63

Dwek, E., \& Scalo, J. M. 1980, ApJ, 239, 193

Dwek, E., Moseley, S. H., Glaccum, W., et al. 1992, ApJ, 389, L21

Dwek, E., Staguhn, J. G., Arendt, R. G., et al. 2011, ApJ, 738, 36

Dwek, E., Staguhn, J., Arendt, R. G., et al. 2014, ApJ, 788, L30

Ennis, J. A., Rudnick, L., Reach, W. T., et al. 2006, ApJ, 652, 376

Fesen, R. A., Morse, J. A., Chevalier, R. A., et al. 2001, AJ, 122, 2644

Fesen, R. A., Hammell, M. C., Morse, J., et al. 2006, ApJ, 636, 859

Fesen, R. A., Zastrow, J. A., Hammell, M. C., Shull, J. M., \& Silvia, D. W. 2011 ApJ, 736, 109

Gall, C., Andersen, A. C., \& Hjorth, J. 2011a, A\&A, 528, A13

Gall, C., Andersen, A. C., \& Hjorth, J. 2011b, A\&A, 528, A14

Gall, C., Hjorth, J., \& Andersen, A. C. 2011c, A\&ARv, 19, 43

Ghavamian, P., Laming, J. M., \& Rakowski, C. E. 2007, ApJ, 654, L69

Gomez, H. L., Krause, O., Barlow, M. J., et al. 2012, ApJ, 760, 96

Gotthelf, E. V., Koralesky, B., Rudnick, L., et al. 2001, ApJ, 552, L39

Hammell, M. C., \& Fesen, R. A. 2008, ApJS, 179, 195

Helder, E. A., \& Vink, J. 2008, ApJ, 686, 1094

Hester, J. J. 2008, ARA\&A, 46, 127

Hwang, U., \& Laming, J. M. 2009, ApJ, 703, 883

Hwang, U., \& Laming, J. M. 2012, ApJ, 746, 130

Indebetouw, R., Matsuura, M., Dwek, E., et al. 2014, ApJ, 782, L2

Itoh, H. 1981, PASJ, 33, 521

Kamper, K., \& van den Bergh, S. 1976, ApJS, 32, 351

Klein, R. I., McKee, C. F., \& Colella, P. 1994, ApJ, 420, 213

Krause, O., Birkmann, S. M., Usuda, T., et al. 2008, Science, 320, 1195

Lagage, P. O., Claret, A., Ballet, J., et al. 1996, A\&A, 315, L273

Laming, J. M., \& Hwang, U. 2003, ApJ, 597, 347

Mathis, J. S., Rumpl, W., \& Nordsieck, K. H. 1977, ApJ, 217, 425

Matsuura, M., Dwek, E., Barlow, M. J., et al. 2015, ApJ, 800, 50

McKee, C. F. 1974, ApJ, 188, 335

Micelotta, E. 2013, Oral presentation at the conference: The Life Cycle of Dust in the Universe: Observations, Theory, and Laboratory Experiments (LCDU2013), 18-22 November, Taipei, Taiwan,

http://eventz.asiaa.sinica.edu.tw/meeting/20131118/talk/ 2013112211_Talk_ElisabettaMicelotta.pdf

Micelotta, E., \& Dwek, E. 2013, in Proc. Life Cycle of Dust in the Universe: Observations, Theory, and Laboratory Experiments (LCDU2013), 18-22 November, Taipei, Taiwan, eds. A. Andersen, M. Baes, H. Gomez, C. Kemper, \& D. Watson 
Morse, J. A., Fesen, R. A., Chevalier, R. A., et al. 2004, ApJ, 614, 727

Moseley, S. H., Dwek, E., Glaccum, W., Graham, J. R., \& Loewenstein, R. F. 1989, Nature, 340, 697

Nath, B. B., Laskar, T., \& Shull, J. M. 2008, ApJ, 682, 1055

Nozawa, T., Kozasa, T., Umeda, H., Maeda, K., \& Nomoto, K. 2003, ApJ, 598, 785

Nozawa, T., Kozasa, T., \& Habe, A. 2006, ApJ, 648, 435

Nozawa, T., Kozasa, T., Habe, A., et al. 2007, ApJ, 666, 955

Nozawa, T., Kozasa, T., Tominaga, N., et al. 2010, ApJ, 713, 356

Peimbert, M., \& van den Bergh, S. 1971, ApJ, 167, 223

Reed, J. E., Hester, J. J., Fabian, A. C., \& Winkler, P. F. 1995, ApJ, 440, 706

Rho, J., Kozasa, T., Reach, W. T., et al. 2008, ApJ, 673, 271

Rho, J., Jarrett, T. H., Reach, W. T., Gomez, H., \& Andersen, M. 2009, ApJ, 693, L39

Rho, J., Onaka, T., Cami, J., \& Reach, W. T. 2012, ApJ, 747, L6

Schneider, R., Ferrara, A., \& Salvaterra, R. 2004, MNRAS, 351, 1379

Silvia, D. W., Smith, B. D., \& Shull, J. M. 2010, ApJ, 715, 1575

Silvia, D. W., Smith, B. D., \& Shull, J. M. 2012, ApJ, 748, 12

Slavin, J. D., Dwek, E., \& Jones, A. P. 2015, ApJ, 803, 7

Sutherland, R. S., \& Dopita, M. A. 1993, ApJS, 88, 253

Sutherland, R. S., \& Dopita, M. A. 1995, ApJ, 439, 381

Temim, T., \& Dwek, E. 2013, ApJ, 774, 8

Temim, T., Dwek, E., Tchernyshyov, K., et al. 2015, ApJ, 799, 158
Thorstensen, J. R., Fesen, R. A., \& van den Bergh, S. 2001, AJ, 122, 297

Tielens, A. G. G. M., McKee, C. F., Seab, C. G., \& Hollenbach, D. J. 1994, ApJ, 431, 321

Todini, P., \& Ferrara, A. 2001, MNRAS, 325, 726

Truelove, J. K., \& McKee, C. F. 1999, ApJS, 120, 299

Valiante, R., Schneider, R., Salvadori, S., \& Bianchi, S. 2011, MNRAS, 416, 1916

van Adelsberg, M., Heng, K., McCray, R., \& Raymond, J. C. 2008, ApJ, 689, 1089

van den Bergh, S. 1971, ApJ, 165, 457

van den Bergh, S., \& Kamper, K. 1985, ApJ, 293, 537

Vink, J., Kaastra, J. S., \& Bleeker, J. A. M. 1996, A\&A, 307, L41

Wallström, S. H. J., Biscaro, C., Salgado, F., et al. 2013, A\&A, 558, L2

Watson, D., Christensen, L., Knudsen, K. K., et al. 2015, Nature, 519, 327

Willingale, R., Bleeker, J. A. M., van der Heyden, K. J., Kaastra, J. S., \& Vink, J. 2002, A\&A, 381, 1039

Willingale, R., Bleeker, J. A. M., van der Heyden, K. J., \& Kaastra, J. S. 2003, A\&A, 398, 1021

Wooden, D. H. 1997, in AIP Conf. Ser. 402, eds. E. K. Zinner, \& T. J. Bernatowicz, 317

Yamaguchi, H., Eriksen, K. A., Badenes, C., et al. 2014, ApJ, 780, 136

Young, P. A., Fryer, C. L., Hungerford, A., et al. 2006, ApJ, 640, 891

Zinner, E. 2008, PASA, 25, 7 


\section{Appendix A: Derivation of the equations for the forward and reverse shocks}

The starting point to derive the equation for the blast-wave radius during the initial envelope phase (Eq. (24)) is the first line of Eq. (A.7) in TM99, which we report here for clarity, i.e.

$$
\begin{aligned}
R_{\mathrm{b}}^{*(3-s) / 2}= & (3-s)\left(\frac{\ell_{\mathrm{ED}}}{\phi_{\mathrm{ED}}}\right)^{1 / 2} \\
& \times \frac{f_{n}^{1 / 2}}{3-n}\left[1-\left(\frac{w_{\mathrm{b}}}{\ell_{\mathrm{ED}}}\right)^{(3-n) / 2}\right] \quad w_{\text {core }} \leq\left(\frac{w_{\mathrm{b}}}{\ell_{\mathrm{ED}}}\right) \leq 1,
\end{aligned}
$$

where $w_{\mathrm{b}} \equiv\left[R_{\mathrm{b}}(t) / t\right] / v_{\mathrm{ej}}$ and the term in squared brackets represents the velocity of the free expanding ejecta at a radius equal to $R_{\mathrm{b}}$. Using Eqs. (4) and (12) we can rewrite $w_{\mathrm{b}}$ in terms of starred variables as follows:

$w_{\mathrm{b}}=\frac{R_{\mathrm{b}}^{*} / t^{*}}{v_{\mathrm{ej}}^{*}}=\left(\frac{\bar{\alpha}}{2}\right)^{1 / 2} \frac{R_{\mathrm{b}}^{*}}{t^{*}}$.

Substituting Eq. (A.2) in Eq. (A.1) we can derive Eq. (A.8) in TM99, which expresses $t^{*}$ as a function of $R_{\mathrm{b}}^{*}$ (below),

$$
\begin{aligned}
t^{*}\left(R_{\mathrm{b}}^{*}\right)= & \left(\frac{\bar{\alpha}}{2}\right)^{1 / 2} \frac{R_{\mathrm{b}}^{*}}{\ell_{\mathrm{ED}}} \\
& \times\left[1-\left(\frac{3-n}{3-s}\right)\left(\frac{\phi_{\mathrm{ED}}}{\ell_{\mathrm{ED}} f_{n}}\right)^{1 / 2} R_{\mathrm{b}}^{*(3-s) / 2}\right]^{-2 /(3-n)} .
\end{aligned}
$$

To derive Eq. (24), we consider the case when $w_{\text {core }} \rightarrow 0$. This implies $f_{n} \rightarrow 0$ (see Eq. (11)) and allows us to neglect the term " 1 " within square brackets in Eq. (A.3). For $w_{\text {core }} \rightarrow 0$, Eqs. (11) and (12) lead to

$$
f_{n} \sim \frac{3}{4 \pi} \frac{n-3}{n} w_{\text {core }}^{n-3} \quad \bar{\alpha} \sim \frac{3}{5} \frac{n-3}{n-5} w_{\text {core }}^{2} .
$$

Substituting the above expressions and Eq. (16) in the modified Eq. (A.3), we derive $R_{\mathrm{b}}^{*}\left(t^{*}\right)$ during the envelope phase (Eq. (24)).

To derive the time $t_{\text {core }}^{*}$ at which the reverse shock hits the ejecta core, we used the fact that $v_{\text {core }}^{*}=R_{\mathrm{r}}^{*} / t_{\text {core }}^{*}$ and $R_{\mathrm{r}}^{*}=$ $R_{\mathrm{b}}^{*} / \ell_{\mathrm{ED}}$. This leads to

$$
t_{\text {core }}^{*}=\frac{R_{\mathrm{b}}^{*}}{\ell_{\mathrm{ED}} v_{\text {core }}^{*}} .
$$

Substituting $R_{\mathrm{b}}^{*}$ with the envelope solution at $t^{*}=t_{\text {core }}^{*}$ we obtained Eq. (30) for $t_{\text {core }}^{*}$.

The radius of the blast wave during the core phase is given, in implicit form, by the second line of equation A7 in TM99, which is written as

$$
\begin{aligned}
R_{\mathrm{b}}^{*(3-s) / 2}= & (3-s)\left(\frac{\ell_{\mathrm{ED}}}{\phi_{\mathrm{ED}}}\right)^{1 / 2} \frac{f_{0}^{1 / 2}}{3}\left[w_{\text {core }}^{3 / 2}-\left(\frac{w_{\mathrm{b}}}{\ell_{\mathrm{ED}}}\right)^{3 / 2}\right] \\
& +\frac{f_{n}^{1 / 2}}{3-n}\left(1-w_{\text {core }}^{(3-n) / 2}\right) \quad 0 \leq\left(\frac{w_{\mathrm{b}}}{\ell_{\mathrm{ED}}}\right) \leq w_{\text {core }} .
\end{aligned}
$$

For $s \geq 2$, the derivation of $R_{\mathrm{b}}^{*}$ from Eq. (A.6) is extremely complicated, thus we decided to adopt the same approach as Laming \& Hwang (2003). This procedure extends the blast-wave solution for the envelope phase into the core phase, matching it to the solution that is appropriate in the Sedov-Taylor (ST) limit. During the ST phase, the mass of the swept-up material exceeds $M_{\mathrm{ej}}$ and the remnant expands following a self-similar motion.
The appropriate solution in the ST limit is the offset power-law given in Eq. (A.12) from TM99, i.e.

$$
\begin{aligned}
R_{\mathrm{b}}^{*}\left(t^{*}\right) & =\left[R_{\mathrm{ST}}^{*(5-s) / 2}+\xi_{s}^{1 / 2}\left(t^{*}-t_{\mathrm{ST}}^{*}\right)\right]^{2 /(5-s)} \text { with } \\
\xi_{s} & =\frac{(5-s)(10-3 s)}{8 \pi}
\end{aligned}
$$

which is obtained integrating the classical ST solution from a characteristic radius $R_{\mathrm{ST}}^{*}$ and time $t_{\mathrm{ST}}^{*}$ to the current radius and time, $R_{\mathrm{b}}^{*}$ and $t^{*}$, respectively. The blast-wave velocity (Eq. (27)) comes directly from Eq. (A.7). To derive the time, $t_{\text {conn }}^{*}$ at which the envelope solution and the ST offset power-law solution connect, we equated Eqs. (25) and (27) to derive an expression for $t^{*}$ as a function of $R_{\mathrm{b}}^{*}$. Then, we substituted in this expression the envelope solution for $R_{\mathrm{b}}^{*}$ (Eq. (24)) obtaining Eq. (29) for $t_{\text {conn }}^{*}$. The final expression for the blast-wave radius valid for $t^{*}>t_{\text {conn }}^{*}$ (Eq. (26)), is derived from ST offset power-law solution (Eq. (A.7)) imposing the conditions $R_{\mathrm{ST}}^{*}=R_{\mathrm{b}}^{*}\left(t^{*}=t_{\text {conn }}^{*}\right)$ (where $R_{\mathrm{b}}^{*}$ is the envelope solution) and $t_{\mathrm{ST}}^{*}=t_{\mathrm{conn}}^{*}$, and using for $v_{\text {core }}^{*}$ the expression from Eq. (16).

To calculate the velocity of the reverse shock in the frame of the unshocked ejecta during the envelope phase $\left(t^{*} \leq t_{\text {core }}^{*}\right)$, we start from the following equation:

$v_{\mathrm{r}}^{*}=v^{*}\left(R_{\mathrm{r}}^{*}, t^{*}\right)-\frac{\mathrm{d} R_{\mathrm{r}}^{*}}{\mathrm{~d} t^{*}}$

The first term on the right side of the equation represents the velocity of the freely expanding ejecta when they encounter the reverse shock and equals $R_{\mathrm{r}}^{*} / t^{*}$, while the second term is the velocity of the reverse shock in the rest frame. Using Eqs. (25) and (31), with few manipulations we obtain Eq. (32) for the reverse shock velocity.

Following Laming \& Hwang (2003), we assume that during the core phase $\left(t^{*}>t_{\text {core }}^{*}\right)$ the reverse shock velocity remains constant at the value given by Eq. (32) evaluated for $t^{*}=t_{\text {core }}^{*}$. This leads immediately to Eq. (34).

To derive the radius of the reverse shock during the core phase, we rewrite Eq. (A.8) as follows:

$v_{\mathrm{r}}^{*}=-t^{*} \frac{\mathrm{d}}{\mathrm{d} t^{*}}\left(\frac{R_{\mathrm{r}}^{*}}{t^{*}}\right)$

The solution of the above equation evaluated between $t_{\text {core }}^{*}$ and $t^{*}$ provides the expression for the reverse shock radius $R_{\mathrm{r}}^{*}\left(t^{*}\right)$ (Eq. (33)), where Eq. (26) has to be used for $R_{\mathrm{b}}^{*}$.

\section{Appendix B: A statistical approach to calculate the fraction of dust surviving kinetic sputtering}

We report here an analytical calculation to estimate, for a given grain size, the fraction of dust able to survive kinetic sputtering in the ejecta clouds. This statistical approach considers a normalized distribution of purely geometrical column densities, while the velocity dependency, which is related to the particle size, is given by Eq. (48).

Figure B.1 is a cross section of a sphere of radius $R$ representing an ejecta cloud of Cas A. The shock impinges on the cloud from the right. The grey region in the figure is the locus of all the dust grains that are at a distance $b$ from the cloud's surface. The volume occupied by all the grains at a vertical distance $y$ from the $x$-axis and a distance between $b-\mathrm{d} b / 2$ and $b+\mathrm{d} b / 2$ from the surface is given by the projected volume of $2 \pi y \mathrm{~d} y \mathrm{~d} b$, where $y_{\mathrm{m}}$, the maximum value of $y$ for a given $b$ is 
equal to $\overline{O G}=\left[R^{2}-(b / 2)^{2}\right]^{1 / 2}$. The differential volume $\mathrm{d} V(b)$ is then

$\mathrm{d} V(b)=\mathrm{d} b \int_{0}^{y_{\mathrm{m}}} 2 \pi y \mathrm{~d} y=\pi\left[R^{2}-(b / 2)^{2}\right]$.

The distribution of distances, normalized to the volume of the cloud is then given by

$\frac{1}{V} \frac{\mathrm{d} V(b)}{\mathrm{d} b}=\frac{3}{4} \frac{R^{2}-b^{2} / 4}{R^{3}}$

The average column density $\langle b\rangle$ is

$\langle b\rangle=\frac{3}{4} \int_{0}^{2 R}\left(\frac{R^{2}-b^{2} / 4}{R^{3}}\right) b \mathrm{~d} b=\frac{3}{4} R$.

The column density, $N$, traversed by a dust grain until it reaches the clouds's surface is given by $N=n_{\text {gas }} b$, where $n_{\text {gas }}$ is the gas density. So the normalized distribution of column densities is given by

$\frac{\mathrm{d} f(N)}{\mathrm{d} N}=\frac{3}{2} \frac{N_{\mathrm{k}}^{2}-N^{2}}{N_{\mathrm{k}}^{3}}$,

where $N_{\mathrm{k}}=2 n_{\mathrm{gas}} R$ is the column density through the centre of the cloud.

We let $f_{\mathrm{M}}\left(v_{\mathrm{gr}}(0), N\right) \equiv m_{\mathrm{gr}}(t) / m_{\mathrm{gr}}(0)$ be the fraction of surviving dust, with an initial velocity $v_{\mathrm{gr}}(0)$ after it traversed a columnn density $N$. The mass fraction of the dust in a cloud that survives the passage of the reverse shock, $\xi\left[v_{\text {cloud }}(\alpha)\right]$ is given by the integral

$\xi\left[v_{\text {cloud }}(\alpha)\right]=\int_{0}^{N_{\mathrm{k}}} f_{\mathrm{M}}\left(v_{\text {cloud }}(\alpha), N\right) \frac{\mathrm{d} f(N)}{\mathrm{d} N}$,

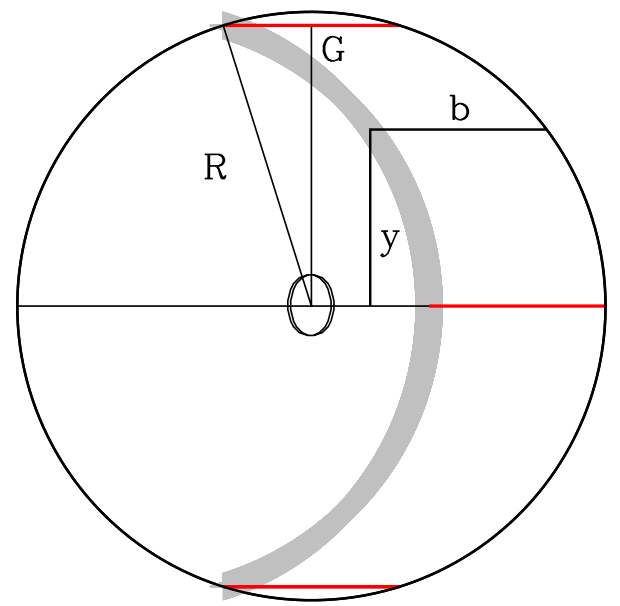

Fig. B.1. Cross section of a spherical ejecta cloud of radius $R$ in Cas A. The grey area is the locus of all points in the cloud that are at the same distance $b$ (represented by the red lines) from the surface of the cloud.

where $v_{\text {cloud }}(\alpha)$ is the velocity of the cloud shock through a clump located in the ejecta layer $\alpha$, and where we assumed that because of the strong compression, the reverse shock gives all grains an initial velocity $v_{\text {cloud }}(\alpha)$, relative to the gas.

The statistical approach described above is only applicable for the largest grain sizes, for which the maximum column density through the clump, $2 n_{\text {gas }} R$ is smaller than that required to slow them down to the kinetic sputtering threshold. For smaller grain sizes, a Monte Carlo approach, as described in Sect. 7, is needed to determine the actual amount of kinetic sputtering before the grain exits the clump, or before the clump is destroyed by the reverse shock. 